\title{
POSTPRINT
}

\section{Detecting conditional healthiness of food items from natural language text}

\author{
Michael Wiegand ${ }^{1}$ \\ Dietrich Klakow ${ }^{1}$
}

\begin{abstract}
In this article, we explore the feasibility of extracting suitable and unsuitable food items for particular health conditions from natural language text. We refer to this task as conditional healthiness classification. For that purpose, we annotate a corpus extracted from forum entries of a food-related website. We identify different relation types that hold between food items and health conditions going beyond a binary distinction of suitability and unsuitability and devise various supervised classifiers using different types of features. We examine the impact of different task-specific resources, such as a healthiness lexicon that lists the healthiness status of a food item and a sentiment lexicon. Moreover, we also consider task-specific linguistic features that disambiguate a context in which mentions of a food item and a health condition co-occur and compare them with standard features using bag of words, part-of-speech information and syntactic parses. We also investigate in how far individual food items and health conditions correlate with specific relation types and try to harness this information for classification.
\end{abstract}

Keywords: Text classification, Food domain, Social media, Linguistically informed feature engineering, Polarity classification

\section{Introduction}

Food plays a substantial part in each of our lives. This is not only due to the fact that we need it to survive but also since it has social and cultural significance. With the growing health awareness in many parts of the population, there is consequently a

Michael Wiegand

michael.wiegand@1sv.uni-saarland.de

1 Spoken Language Systems, Saarland University, Building C7.1, 66123 Saarbrücken, Germany 
high demand for the knowledge about the tolerability of different food items for specific health conditions. In view of the variety of both different types of food and health conditions it does not come as a surprise that there does not exist a common repository summarizing all that knowledge. Since, however, much of this information is preserved in natural language text, we assume that it is possible to acquire some of this knowledge automatically with the help of natural language processing.

In this article, we examine the task of identifying mentions that a food item is suitable (1) or unsuitable (2) given a particular health condition. We will also refer to this type of classification as conditional healthiness classification. Therefore, Sentence (1) is considered an example of conditional healthiness while Sentence (2) is considered an example of conditional unhealthiness.

(1) People suffering from gout may eat tomatoes.

(2) During pregnancy women should not consume any alcohol.

Instead of just considering the binary distinction between suitability and unsuitability, we will consider typical subclasses. For example, a suitable food item may also alleviate the symptoms of the health condition (3) or even prevent the disease from breaking out (4).

(3) Ginger is very good for your stomach-it helped me a lot against my heartburn.

(4) Iron deficiency can usually be prevented by consuming meat on a regular basis.

To the best of our knowledge, this is the first work that addresses this type of classification. Therefore, we need to answer some basic questions: we want to know whether this type of information can be extracted from natural language text at all, what relation types occur sufficiently often so that automatic extraction is possible, and what types of features for supervised classifiers should be taken into consideration. Apart from standard bag-of-words features, we consider a set of linguistic features. In addition, we will also test some manually compiled keywords collected from our dataset. Thus, we have an upper bound estimate in how far lexical information would help to distinguish between the different classes.

Moreover, we want to investigate in how far natural language processing is beneficial in the presence of simple heuristics. A straightforward baseline would be, for instance, to always classify a food item according to general healthiness (which is usually derived from general nutrient content) and thus completely ignore specific health conditions. For example, since apples are generally considered healthy, they would also be predicted as conditional healthy food items. In addition, we are interested in how far certain health conditions correlate with particular class labels. If there is generally a strong correlation between a particular health condition and a particular class label, e.g. diarrhoea and causation, ${ }^{1}$ then classifying food items for a particular health condition from natural language text could be reduced to just

\footnotetext{
${ }^{1}$ In our dataset, people mostly discuss food items that cause the outbreak of that disease rather than food items that protect them against it or alleviate the symptoms if they contract it.
} 
extracting food items that frequently co-occur with mentions of that condition. (The class label would always be the one that mostly co-occurs with the respective health condition.) Further contextual disambiguation would be superfluous.

Note that we are exclusively interested in the classification of individual utterances rather than an aggregate assessment of a set of utterances. However, it should be noted that the former is a pre-requisite of the latter: it is not possible to produce an aggregate assessment without a correct assessment of (most) individual utterances.

In terms of applications, the task we examine in this article could be seen as a further step towards a more intelligent search engine, that is, a search engine being able to retrieve content not only on the basis of keyword matching but also on the basis of some limited form of text understanding. Websites that host large user forums on food and/or health-related issues, as the one from which we extracted our gold standard (see also Sect. 2), could incorporate such technology in their search inferface allowing their users a more focused search.

Our experiments are carried out on German data. We believe, however, that our findings carry over to other languages since the linguistic aspects that we address are (mostly) language universal. For the sake of general accessibility of this article, all German examples will be accompanied by English translations.

The major contributions of this article are (i) a new annotation scheme for conditional healthiness classification, (ii) a comparison of diverse features for supervised classification and (iii) a detailed error analysis in which we try to uncover the reasons why certain features are not effective or why they only produce low classification performance for certain classes.

\section{The dataset}

In order to generate a dataset for our experiments, we used a crawl of chefkoch.de $e^{2}$ (Wiegand et al. 2012b) consisting of 418.558 webpages of food-related forum entries. chefkoch.de is the largest web portal for food-related issues in the German language. In Wiegand et al. (2012a, 2014) it was found that for domain-specific relation extraction in the food domain, such a corpus largely outperforms alternative open-domain data collections, such as Wikipedia.

The advantage of using a domain-specific corpus is that we find much more content for the target relations than in other standard corpora. For instance, we found that our domain-specific corpus contains almost 5 times as many cooccurrences of a food item and a health condition (in one sentence) ${ }^{3}$ as the German Wikipedia dump created at (roughly) the same time our domain-specific food corpus was created. Compared with the German subset of the Web $1 T$ gram

\footnotetext{
2 www.chefkoch.de.

${ }^{3}$ Note that co-occurrences of a food item and a health condition are only an approximation of genuine food-health relations. In other words, not every of these co-occurrences necessarily conveys a proper food-health relationship. However, without manually annotating each of these co-occurrences, this is the best approximation we can produce.
} 
(LDC2009T25), ${ }^{4}$ our domain-specific corpus contains even 6 times as many cooccurrences. ${ }^{5}$

While we are aware of the fact that the conditional healthiness of food items is also discussed in scientific (medical) texts, these types of text (with a size similar to our web corpus) are not available to us. (In fact, we believe that it is fairly difficult to acquire a corpus from the medical domain with the same diversity of cooccurrences of health conditions and food items. This is due to the fact that individual medical texts are more thematically focused. That is, they typically just deal with a particular pair of health condition and food item, e.g. eggs and heart disease.) However, we do not only examine this classification task on a web corpus just because other text types are not available to us. We think that the text analysis on web data serves its own purpose. Scientific medical texts are due to their complexity hardly accessible to people without solid background knowledge in medical science. On the other hand, conditional healthiness attracts a large part of the general population. The forum entries we extracted employ a language that is much easier to follow. Thus, we believe that text excerpts from forum entries are more suitable to satisfy the information need of that part of the population.

Not only do medical texts differ from social media in the type of language, they also differ in the type of content they provide. Social media can be considered as an exclusive repository of popular wisdom. With regard to the health conditions, we can find, for example, home remedies. Despite the fact that many of them are not scientifically proven, there is still a great interest in that type of knowledge. In this work, we do not make any attempts to separate genuine facts from claims. The majority of relations extracted are just unsubstantiated claims made by the public.

\subsection{The choice of health conditions}

Table 1 lists the health conditions we consider for the creation of our dataset. We focused on those health conditions where at least a mild relationship between the condition and food (in general) is known. This is ensured by the fact that the list is a subset of conditions for which suitable and unsuitable food items have been listed in the gold standard introduced in Wiegand et al. (2012c). Another side-effect of restricting ourselves to entries from this gold standard is that these health conditions occur fairly frequently (for an average health condition we find 64 mentions in our dataset).

\subsection{Annotation}

Our final dataset consists of 2604 instances, where each instance represents a sentence in which a food item co-occurs with one of the health conditions. While we restrict the health conditions to the ones listed in Table 1, we make no restriction on

\footnotetext{
${ }^{4}$ For this corpus, we can only observe food-health co-occurrences within 5-grams and not entire sentences.

${ }^{5}$ The low coverage on the 5-grams can be partially explained by the fact that this corpus only contains ngrams observed at least 40 times. We assume that there are considerably more co-occurrences of food items and health conditions among web-based 5-grams at lower frequencies.
} 
Table 1 List of health conditions (English translation/frequency) for which suitable/unsuitable food items are to be extracted

\title{
Health Conditions
}

\begin{abstract}
Abwehrkräfte (healthy immune system/2), Akne (acne/15), Asthma (asthma/20), Blähungen (flatulence/ 1), Blasenentzündung (cystitis/32), Bluthochdruck (hypertension/32), Depression (depression/19), Diabetes (diabetes/134), Durchfall (diarrhoea/255), Eisenmangel (iron deficiency/29), Eiweißallergie (protein intolerance/6), Fieber (fever/127), Gicht (gout/34), Grippe (flu/86), Halsschmerzen (sore throat/183), Heiserkeit (hoarseness/14), Herzkrankheit (heart disease/24), Heuschnupfen (hay fever/8), Husten (cough/191), Kalziummangel (acalcinosis/5), Karies (caries/25), Kondition (body condition/4), Kopfschmerzen (headache/280), Magengeschwür (stomach ulcer/9), Mandelentzündung (tonsillitis/ 11), Müdigkeit (lassitude/44), Mumps (mumps/1), Neurodermitis (dermatitis/194), Nierensteine (kidney stones/16), Reizdarm (irritable bowel syndrome/4), Rheuma (rheumatism/29),

Salmonellenvergiftung (salmonella/17), Schnupfen (coryza/86), Schuppenflechte (psoriasis/9), Schwangerschaft (pregnancy/218), Sodbrennen (heartburn/232), Übelkeit (nausea/103), Übergewicht (overweight/79), Untergewicht (underweight/13), Verstopfung (constipation/75), Vitamin C Mangel (lack of vitamin C/1), Zahnschmerzen (toothache/39)
\end{abstract}

the target food items we consider. Similar to Wiegand et al. (2012a) and Wiegand et al. (2012b), food items are detected with the help of GermaNet (Hamp and Feldweg 1997), the German version of WordNet (Miller et al. 1990).

Table 2 illustrates a typical instance. Note that for one instance, we only specify one target food item and one health condition. This means that in case a sentence contains more than one of these expressions, it may appear several times as a target sentence but each time we will assign a different target food item or health condition.

Each instance of the extracted corpus was manually assessed. Two native speakers of German were employed as annotators. We initially selected three health conditions for which we extracted all sentences in which there is a co-occurrence with some food item. Each of these instances was annotated by both annotators in order to measure interannotation agreement. (The remaining instances were only annotated by one annotator each.) This resulted in 276 instances. For each instance, the annotators were to choose exactly one category label. We measured an interannotation agreement of Cohen's $\kappa=0.7651$. This agreement can be interpreted as substantial (Landis and Koch 1977) and should be sufficiently high for our experiments. The dataset, including the annotation guidelines we devised, will be made publicly available for research purposes.

\subsection{The annotation scheme}

We now describe the different category labels. Their distribution is depicted in Table $3 .^{6}$

\subsubsection{Suitable (SUIT)}

SUIT encompasses all those statements in which the consumption of the target food item is suitable for people affected by a particular health condition (5). By suitable,

\footnotetext{
${ }^{6}$ In the following, we may also refer to category labels as classes or relation types.
} 
Table 2 Illustration of a data instance; target food item: tea, target health condition: heartburn, relation type: CAUSE (Sect. 2.3.6)

\begin{tabular}{ll}
\hline Preceding context & $\begin{array}{l}\text { Ich trinke auch keinen Kaffee, mag auch nicht so gern Tee. } \\
\text { (I don't drink any coffee and I don't like tea very much, either.) }\end{array}$ \\
Preceding context & $\begin{array}{l}\text { Mal welchen, wenn ich krank bin. } \\
\text { (I only drink it when I'm ill.) }\end{array}$ \\
Target sentence & $\begin{array}{l}\text { Aber ich bekomme vom Tee } e_{\text {food_item }} \text { normalerweise Sodbrennen } \\
\text { (But, I usually_condition } .\end{array}$ \\
Succeeding context & $\begin{array}{l}\text { Am liebsten mag ich ganz normales Wasser. } \\
\text { (I prefer drinking mineral water.) }\end{array}$ \\
Succeeding context & $\begin{array}{l}\text { Das ist auch immer so, wenn ich woanders eingeladen bin. } \\
\text { (That's also what I choose when I'm invited somewhere.) }\end{array}$ \\
\hline
\end{tabular}

Table 3 Statistics of the different category labels

\begin{tabular}{llll}
\hline Type & Abbreviation & Frequency & Percentage \\
\hline No relation & NOREL & 539 & 20.70 \\
Beneficial & BENEF & 502 & 19.28 \\
Causation & CAUSE & 482 & 18.51 \\
Suitable & SUIT & 428 & 16.44 \\
Embedded relation & EMBREL & 302 & 11.60 \\
Unsuitable & UNSUIT & 247 & 9.49 \\
Prevention & PREVENT & 74 & 2.84 \\
Worsening & WORSEN & 30 & 1.15 \\
\hline
\end{tabular}

we mean that there will not be a negative effect on the health of a person once he or she consumes the target food item. However, this relation type does not state that the consumption is likely to improve the condition of the person either, even though it is not impossible. The way how the suitability is expressed does not explicitly address positive effects.

(5) Ich hatte auch Neurodermitis, daher verwendete meine Mutter nur Dinkelmehl (anstatt Weizenmehl); man merkt fast keinen Unterschied.

(I also had dermatitis which is why my mother used spelt flour (instead of wheat flour); you can taste almost no difference.)

\subsubsection{Beneficial (BENEF)}

While SUIT only states that the consumption of the target food item is suitable for people with a particular health condition, BENEF actually states that the consumption alleviates the symptoms of the condition or even cures it (6). 
(6) Wenn ich Halsschmerzen bekomme, dann trinke ich immer Milch, das lindert die Schmerzen.

(Usually, a glass of milk helps me when I got a sore throat.)

\subsubsection{Prevention (PREVENT)}

PREVENT presents an even stronger positive effect than the relation type BENEF. It claims that the consumption of the target food item can prevent the outbreak of a particular disease (7).

(7) Der Bildung von Nierensteinen kann durch Zitronensäure vorgebeugt werden. (Citric acid reduces the chances of kidney stones developing.)

\subsubsection{Unsuitable (UNSUIT)}

UNSUIT can be considered the negative counterpart of SUIT. It describes cases in which the consumption of the target food item is deemed unsuitable. Unsuitability means that one expects a negative effect, that is, a deterioration of the health situation on the part of the person who suffers from a particular health condition (8). However, there is no explicit mention that there is some deterioration or how the deterioration manifests itself. Typically, the speaker just advises against the consumption of the target food item given a particular health condition.

(8) Eier sollten während der Schwangerschaft nicht gegessen werden.

(Eggs should not be eaten during pregnancy.)

\subsubsection{Worsening (WORSEN)}

WORSEN is the negative counterpart of BENEF. This relation type explicitly says that the consumption of the target food item results in a deterioration (9). Thus, utterances of that type are perceived to be more intense than utterances of type UNSUIT. The type of deterioration may also be mentioned.

(9) Da bringt Rotwein bei mir den Heuschnupfen so richtig zur Geltung.

(If I now drink red wine, this will heavily increase the symptoms of my hay fever.)

\subsubsection{Causation (CAUSE)}

CAUSE is the negative counterpart of PREVENT. It states that the consumption of the target food item can actually cause a particular health condition (10).

(10) Es ist allgemein bekannt, dass Cola Karies verursacht. (It's a common fact that the regular consumption of coke causes caries.) 


\subsubsection{Embedded relations (EMBREL)}

EMBREL describes cases in which one of the previous six relation types are embedded in a context so that one cannot conclude that this relation type holds. Typical embeddings are questions (11), irrealis (12) or irony (13).

(11) Weiß jemand, wie das mit Tofu und Schwangerschaft ist? (Does anyone know whether I can eat tofu during my pregnancy?)

(12) Wenn man von Schokolade Akne bekäme, würde ich aufhören, sie zu essen. (If chocolate caused acne, I would stop eating it.)

(13) Vom Pute essen wird man schon keine Kopfschmerzen kriegen, da sie mit Schmerzmitteln vollgepumpt werden.

(Eating turkey hen will prevent you from getting a headache, as they drugged them up to the eyeballs.)

Negated relations are only labeled as EMBREL if they cannot be resolved as another unnegated relation type. For instance, if a sentence expresses a negated relation of type SUIT (14), then this can in most cases be translated as an unnegated case of UNSUIT (15).

(14) Ich finde, Du solltest mit Deiner Diabetes keinen Kuchen essen.

(I don't think that you should eat cake with your diabetes.)

(15) Ich finde, dass Kuchen für Dich mit Deiner Diabetes ungeeignet ist.

(I think that cake is unsuitable for you with your diabetes.)

However, a negated case of CAUSE (16), for example, cannot be associated with any other existing category.

(16) Du wirst von Eiern schon kein Durchfall bekommen (allerdings wenn Du ihn bereits hast, würde ich Dir vom Verzehr abraten).

(You won't get diarrhoea from eating eggs (but if you contracted it I wouldn't recommend eating them either).)

If the interrelation between target food item and health condition is expressed with some degree of uncertainty (17); the relation involves an additional restriction, for example with regard to the quantity (18); or the relation is reported by the speaker but there is no definite indication that they share that view (19), then this is not considered as a type of embedding. (In other words, Sentences (17) and (18) would still be labeled CAUSE while Sentence (19) would be labeled UNSUIT.) This is due to the fact that the majority of interrelations are fairly weak. Usually, they are only observed with a smaller number of people. As a consequence of that, it is quite rare that a speaker presents the interrelation between a food item and a particular health condition as a definite fact (that always holds).

(17) Von rotem Fleisch kann man Herzkrankheiten bekommen. (Eating red meat may cause heart diseases.)

(18) Wenn man viel Alkohol trinkt, verursacht das Bluthochdruck. (Drinking a lot of alcohol causes hypertension.) 
(19) Irgendwo habe ich mal gelesen, dass Obst bei Diabetes nicht empfohlen wird.

(I've read somewhere that fruit is not recommended for people suffering from diabetes.)

\subsubsection{No relation (NOREL)}

While in all previously discussed cases the target food item and health condition are somehow related, there are cases in which the co-occurrence is merely co-incidental (20). On our dataset, this is actually the most frequent category (Table 3).

(20) Sein Problem ist gar nicht so sehr die Diabetes, ich mache mir eher Sorgen um das Fett (das er zu sich nimmt).

(It's not his diabetes I'm concerned about but the enormous amounts of fat (that he consumes).)

Finally, Table 4 displays a set of relations our dataset contains. We deliberately also include less common relations in order to support our claim that the textual source we chose for this task is suitable for knowledge acquisition. If our dataset only contained typical relations that are common knowledge-by that we mean relations, such as CAUSE(sugar, caries), BENEF(chicken broth, flu) or UNSUIT(alcohol, pregnancy) — this would be hardly convincing.

Table 4 Some example relations (food item, health condition) from the gold standard illustrating the wide knowledge contained in our text corpus

\begin{tabular}{|c|c|}
\hline Relation Type & Instances \\
\hline SUIT & $\begin{array}{l}\text { (Mandeln/almonds, Neurodermitis/dermatitis); (Lamm/lamb, Neurodermitis/dermatitis); } \\
\text { (Frischkornbrei/fresh grain porridge, Schwangerschaft/pregnancy); (Stevia/stevia, } \\
\text { Diabetes/diabetes); (Kartoffelpüree/mashed potatoes, Halsschmerzen/sore throat) }\end{array}$ \\
\hline BENEF & $\begin{array}{l}\text { (Kaugummi/chewing gum, Sodbrennen/heartburn); (Kokosflocken/coconut flakes, } \\
\text { Sodbrennen/heartburn); (Grünkohl/green cabbage, Kalziummangel/acalcinosis); } \\
\text { (Ingwer/ginger, Übelkeit/nausea); (Pflaumensaft/prune juice, } \\
\text { Verstopfung/constipation) }\end{array}$ \\
\hline PREVENT & $\begin{array}{l}\text { (Zitronensäure/citric acid, Nierensteine/kidney stones); (Grapefruit/grape fruit, } \\
\text { Diabetes/diabetes); (Fenchel/fennel, Calciummangel/acalcinosis); (Vollkornprodukte/ } \\
\text { whole grain products, Karies/caries) }\end{array}$ \\
\hline UNSUIT & $\begin{array}{l}\text { (Mohn/poppy seeds, Schwangerschaft/pregnancy); (Lauch/leek, Schwangerschaft/ } \\
\text { pregnancy); (Rhababer/rhubarb, Rheuma/rheumatism); (Ingwer/ginger, Fieber/fever); } \\
\text { (Dinkel/spelt, Durchfall/diarrhoea) }\end{array}$ \\
\hline WORSEN & $\begin{array}{l}\text { (Milch/milk, Husten/cough); (Kaffee/coffee, Blasenentzündung/cystitis); } \\
\text { (Schokolade/chocolate, Neurodermitis/dermatitis); (Rotwein/red wine, } \\
\text { Schnupfen/coryza); (Kaffee/coffee, Akne/acne) }\end{array}$ \\
\hline CAUSE & $\begin{array}{l}\text { (Hefe/yeast, Sodbrennen/heartburn); (Safran/saffron, Kopfschmerzen/headache); (Honig/ } \\
\text { honey, Asthma/asthma); (Holunderbeere/elderberry, Durchfall/diarrhoea); (Früchtetee/ } \\
\text { fruit-infused tea, Sodbrennen/heartburn); (Flohsamen/psyllium, } \\
\text { Verstopfung/constipation) }\end{array}$ \\
\hline
\end{tabular}


Table 5 Division of coarse-grained classes into fine-grained classes

\begin{tabular}{ll}
\hline Coarse-grained Class & Fine-grained Classes \\
\hline Conditional Healthy & SUIT, BENEF, PREVENT \\
Conditional Unhealthy & UNSUIT, WORSEN, CAUSE \\
\hline
\end{tabular}

\subsection{Coarse-grained classification}

In the beginning of this article, we introduced the two main categories, conditional healthiness and conditional unhealthiness. Table 5 lists the fine-grained (sub)classes for each of these categories that have just been defined in the previous sections. In this article, we will focus on extracting instances of the fine-grained classes. We think that the different fine-grained categories belonging to the same main categories possess quite different properties that require different kinds of features. By focusing on the main categories these different properties would be less obvious.

\section{Feature design}

In the following, we will discuss the different features we employ in this work. The features are divided in the following groups: word-based features (Sect. 3.1), taskspecific linguistic features (Sect. 3.2), generic linguistic features (Sect. 3.3), sentiment features (Sect. 3.4), features derived from a healthiness lexicon (Sect. 3.5), food and health condition priors (Sect. 3.6) and manually extracted keywords (Sect. 3.7).

\subsection{Word-based features}

We use simple bag-of-words features. The words that we encode are the words between the mention of the target food item and the target health condition, and the words immediately preceding and following these two expressions.

\subsection{Task-specific linguistic features}

The task-specific linguistic features we use are listed in Table 6. The initial linguistic features in that table are configurational cues that are mostly designed to indicate whether there is some relationship between target food item and health condition. The co-occurrence within the same clause is usually a good predictor. There are three features to establish this property. While scope operates on the syntactic parse output, ${ }^{7}$ boundary and otherFood are determined on the token level. The quality of parsing is likely to be affected by the heavy noise in our data (forum entries often contain spelling and grammar mistakes and tend to be fragmented), so

\footnotetext{
${ }^{7}$ We adopt the definition of semantic scope from Wiegand and Klakow (2010).
} 


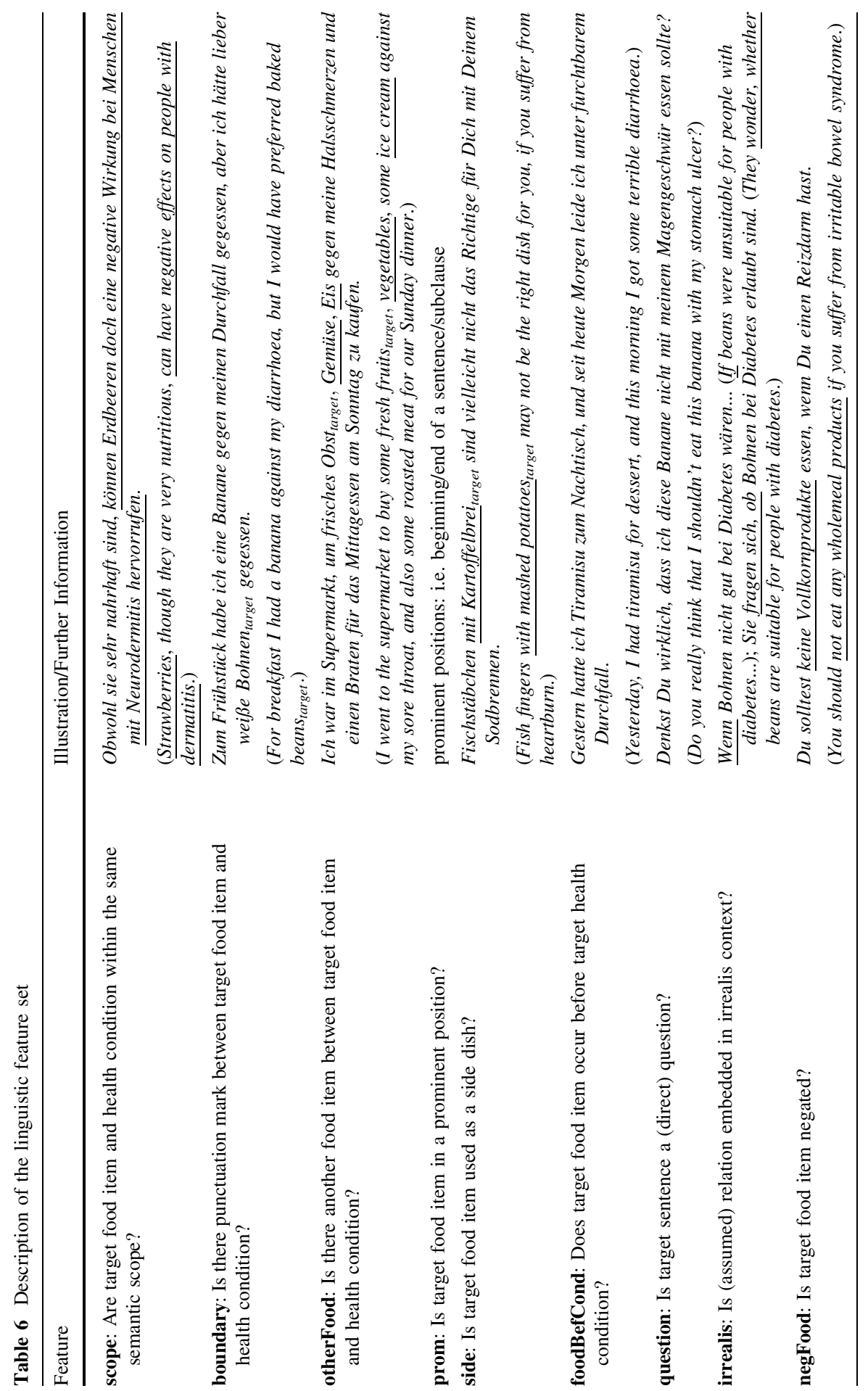




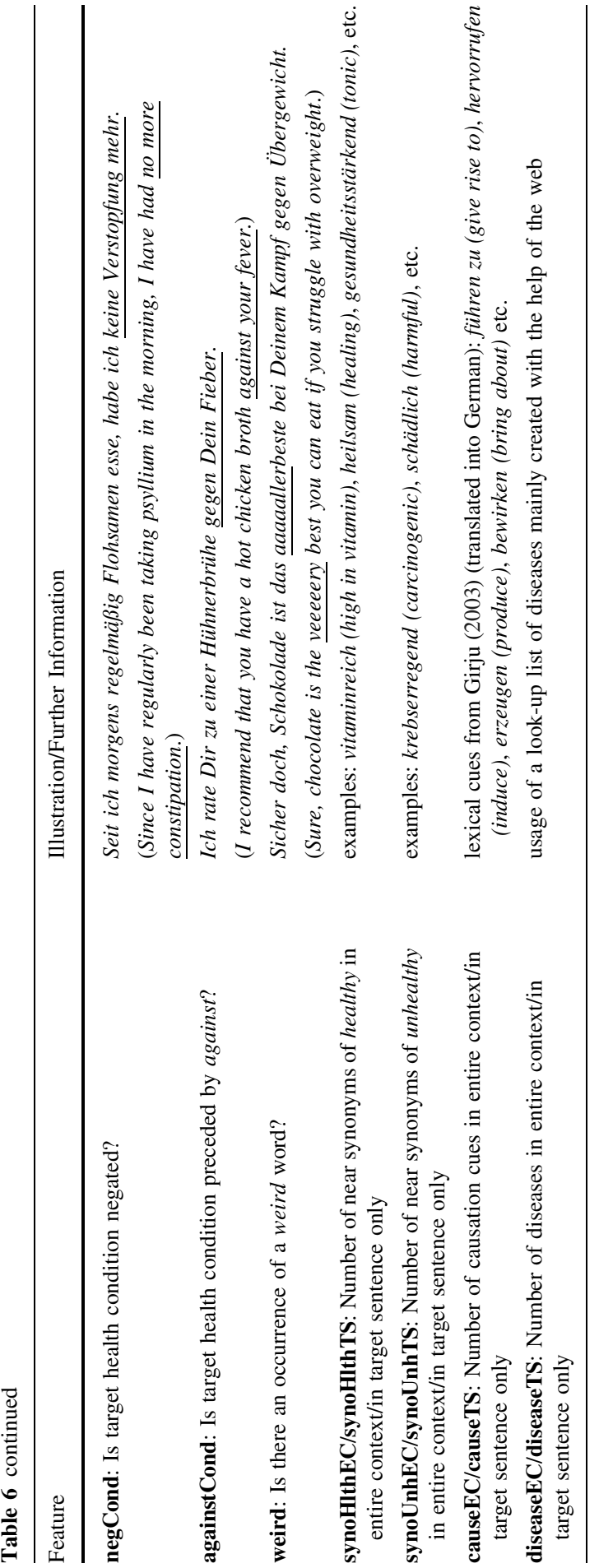


some back-off features (i.e. boundary and otherFood) may be needed. With the feature prom we want to investigate whether the target food item is more likely to be involved in a relation if it is in a prominent sentence position, e.g. the beginning of the sentence. A negative counterpart of prom is side. Possibly, side dishes are less within the focus, so they may be less likely to be involved in a relation. For health conditions, we did not observe similar contexts that would make prominence features (similar to prom and side) necessary. Therefore, we do not include such features for health conditions. foodBefCond takes into consideration the order in which the target food item and health condition appear. This textual order may also reflect the temporal order of events, that is, first a food item is consumed, and then there is some impact on the health condition, e.g. some illness may break out. We do not assume that there is a general correspondence between textual and temporal order, but for some relation types there may be some tendency for a particular textual order.

Table 6 also contains features to detect contextual phenomena that discard the validity of a relation that is embedded. We focus on those types of contextual embeddings that have been presented in Sect. 2.3.7. For the detection of the feature question, we rely on typical surface cues, i.e. ? and the various interrogative pronouns. In order to detect irrealis constructions we scan the target sentence for typical cue words (e.g. wenn (if) or ob (whether)). For negation modeling, i.e. negFood and negCond, we mainly adapted to German the lists of negation words and the scope modeling from Wilson et al. (2005). From a semantic point of view againstCond is used in similar cases as negCond. We insert a pattern with this special construction as it is not captured by the negation words from Wilson et al. (2005). ${ }^{8}$ We assume that the reason for its omission is that this type of construction is very domain or task specific. On our dataset, it is fairly frequent. (It occurs in 180 target sentences.) In order to detect irony, we scan sentences for weird words typically containing sequences of reduplicated characters (e.g. sooooo or seeeeeeehr). This is admittedly only a very shallow feature, but, on the other hand, we only encountered few cases of irony on our dataset.

The features of the third group have in common that they count the frequency of certain types of words. (Unlike the features of the previous group that also made use of look-up lists, these features do not address contextual embedding.) Each of these features comes in two versions. One considers the corresponding cues in the target sentence only (those features carry the suffix $-T S$ ) while another considers the entire context (those features carry the suffix $-E C$ ), that is, in addition to the target sentence it takes into account the two sentences preceding and following the target sentence (Table 2). By this distinction we want to examine which contextual window is most informative for this classification task. synoHlth(EC|TS) and synoUnh(EC|TS) count the number of near synonyms of the word healthy and the word unhealthy. These lexicons were taken from Wiegand and Klakow (2013a). synoHlth(EC|TS) uses 65 cue words while synoUnh(EC|TS) uses 33 cue words. As

\footnotetext{
${ }^{8}$ Negation will also be discussed in our error analysis, particularly in the context of sentiment features (Sect. 5.3) and in the context of the class UNSUIT (Sect. 5.6) (for UNSUIT negation is an important predictor).
} 
Table 7 Description of the generic linguistic features

\begin{tabular}{|c|c|c|}
\hline Subgroup & Feature & Illustration \\
\hline \multirow[t]{5}{*}{ pos } & $\begin{array}{l}\text { part-of-speech sequence between target food item } \\
\text { and health condition }\end{array}$ & APPRART_NN_APPR \\
\hline & part-of-speech tag preceding target food item & APPR $_{\text {beforeFood }}$ \\
\hline & part-of-speech tag following target food item & $\$ \cdot$ afterFood \\
\hline & part-of-speech tag preceding target health condition & VVFIN $_{\text {beforeCond }}$ \\
\hline & part-of-speech tag following target health condition & $\mathrm{APPRART}_{\text {afterCond }}$ \\
\hline path & $\begin{array}{l}\text { path on the syntactic parse tree from target food item } \\
\text { to target health condition }\end{array}$ & $\uparrow \mathrm{NP}_{-} \uparrow \mathrm{PP}_{-} \uparrow \mathrm{NP}_{-} \uparrow \mathrm{PP}_{-} \uparrow \mathrm{VP} \_\downarrow \mathrm{NP}$ \\
\hline
\end{tabular}

Illustration for example sentence: Empfindliche Menschen bekommen Durchfall vom Verzehr von Holunderbeeren. (Some sensitive people get diarrhoea from eating elderberries.)

there have been previous attempts to extract causative relations (Girju and Moldovan 2002; Girju 2003; Beamer and Girju 2009; Kozareva 2012), we could also employ previously introduced lexical resources in order to detect our class CAUSE. We translated the cues from Girju (2003) to German. The resulting lexicon contains 49 expressions. ${ }^{9}$ With regard to the remaining relation types, there do not exist comparable lexical resources we could make use of. Presumably, this is due to the fact that, unlike CAUSE, these are fairly task-specific relation types. Finally, we also count the mentions of diseases (health conditions) in the context of the target food item co-occurring with the target health condition. Possibly, the mention of several diseases in the context of such co-occurrences is indicative of a particular relation type. For the detection of mentions of diseases, we semi-automatically compiled a look-up list mainly relying on the web. In order to have a sufficient coverage on our web forum entries, we made sure that for (very) technical terms, we also have informal expressions (e.g. Grippe ( $f l u$ ) for Influenza (influenza)) that are more likely to be used in every-day language (as can be found in our corpus). The final list of diseases contains 411 expressions.

\subsection{Generic linguistic features}

Apart from the task-specific linguistic features, we will also consider common linguistic features that are often employed in relation extraction tasks. They are listed in Table 7. On the one hand, we consider part-of-speech information (Bunescu and Mooney 2005; Kessler and Nicolov 2009; Mintz et al. 2009). More precisely, we consider the part-of-speech sequence between the target food item and the target health condition. In addition, we also include the part-of-speech tags of the words immediately preceding and following the target expressions. (We do not include the part-of-speech tags of the target food item and the target health condition since, being common nouns, they are always the same. Therefore, nothing

\footnotetext{
9 As there is not a one-to-one mapping between the English keywords from Girju (2003) and their German counterparts, the size of the English and the German lists varies slightly.
} 
can be learnt from that information.) On the other hand, we consider the path on the syntactic parse tree from the target food item to the target health condition (Gildea and Jurafsky 2002; Kessler and Nicolov 2009; Mintz et al. 2009).

\subsection{Features derived from a sentiment lexicon}

The two main category labels, i.e. conditional healthiness and unhealthiness, resemble very much the labels from polarity classification (Wilson et al. 2005). In this subtask of sentiment analysis one needs to distinguish between positive and negative opinions. Both problems (i.e. conditional healthiness and polarity classification) can be considered a binary classification problem comprising two opposing (polar) classes. Due to this similarity, we want to investigate whether lexical cues from polarity classification can be harnessed as features for our task. The cues we consider in this article are so-called polar expressions, i.e. words conveying either positive polarity, such as gut (good), angenehm (pleasant) or herrlich (superb), or negative polarity, such as schlecht (bad), unangenehm (awkward) or schrecklich (horrible). They are obtained from the sentiment lexicon underlying the PolArt system (Klenner et al. 2009). This lexicon has been manually created and contains 7854 polar expressions. The lexicon also assigns an intensity score to each polar expression varying from 0.5 to 1.0 where the latter denotes very strong intensity. For our features we specially mark the strong polar expressions, that is, the expressions that have been assigned a score of 1.0.

The entire set of sentiment features we use is displayed in Table 8. Overall, these features can be divided into two main groups: polarCont comprises features that count the occurrences of different types of polar expressions in the entire context or just the target sentence, while polarSynt is a set of features that considers polar expressions that are (directly) syntactically related to either the target food item or the target health condition. If, for example, the target health condition is a prepositional object of a polar expression, as Zahnschmerzen (toothache) is of Hausrezept (remedy) in Sentence (21), then one or more features from the group polarSynt fire. We add polarSynt in order to find out whether sentiment information combined with syntactic knowledge is actually necessary. Ideally, the combination is more precise than the plain sentiment features from the group polarCont.

(21) Nelken sind ein altes Hausrezept ${ }^{+}$gegen Zahnschmerzen.

(Cloves are an old home remedy ${ }^{+}$against toothache.)

The sentiment features we employ in this article also resemble some of our taskspecific linguistic features (Sect. 3.2), namely synoHlth(ECITS) and synoUn$h(E C \mid T S)$. However, there is some crucial difference between these two types of features: Our sentiment features are derived from a domain-independent lexical resource while the other features are very domain specific. Therefore, the sentiment lexicon with its 7854 entries is much larger than the look-up lists for the taskspecific linguistic features (they comprise 97 entries in total). Despite the difference in size, the domain-specific look-up lists contain only $40 \%$ of the expressions that can also be found in the domain-independent sentiment lexicon. Apparently, there 
Table 8 Features derived from the sentiment lexicon (Klenner et al. 2009)

\begin{tabular}{lll}
\hline Subgroup & Feature & Abbreviation \\
\hline polarCont & Number of positive polar expressions in target sentence only & posTS \\
& Number of negative polar expressions in target sentence only & negTS \\
& Number of strong positive polar expressions in target sentence only & sPosTS \\
& Number of strong negative polar expressions in target sentence only & sNegTS \\
& Number of positive polar expressions in entire context & posEC \\
& Number of negative polar expressions in entire context & negEC \\
& Number of strong positive polar expressions in entire context & sPosEC \\
Number of strong negative polar expressions in entire context & sNegEC \\
Is target food item syntactically related to positive polar expression? & posFoodSynt \\
Is target food item syntactically related to negative polar expression? & negFoodSynt \\
Is target food item syntactically related to strong positive polar expression? & sPosFoodSynt \\
Is target food item syntactically related to strong negative polar & sNegFoodSynt \\
$\quad$ expression? & \\
Is target health condition syntactically related to positive polar expression? & posCondSynt \\
Is target health condition syntactically related to negative polar & negCondSynt \\
expression? & \\
Is target health condition syntactically related to strong positive polar & sPosCondSynt \\
Is target health condition syntactically related to strong negative polar & sNegCondSynt \\
expression? & \\
\hline
\end{tabular}

are some important domain-specific expressions missing in the sentiment lexicon (e.g. krebserregend (carcinogenic) or heilkräftig (medicative)). So, even though the domain-specific features include smaller look-up lists, they may still be effective for our task.

\subsection{Features derived from a healthiness lexicon}

A further group of features incorporates the knowledge of healthiness of food items. This knowledge is obtained from a lexicon introduced in Wiegand et al. (2012c) which covers 2803 food items. Each food item is specified as being either rather or definitely (un)healthy. The healthiness judgment has been carried out based on the general nutrient content of each food item. It has been created totally independently of the annotation of our text corpus. (For a more detailed description of the annotation scheme, we refer the reader to Wiegand et al. (2012c).) Each food item has been annotated by two annotators. Along their individual annotation, a third (adjudicated) annotation has been produced. For our experiments, we use the latter. We consider a food item (un)healthy if it is either classified as rather or definitely (un)healthy. In addition, we also have additional features for definitely (un)healthy food items. 
Even though the healthiness labels that can be found in the healthiness lexicon bear some resemblance to the two main categories, i.e. conditional healthiness and unhealthiness (Sect. 2.4), they are not the same. The healthiness lexicon does not encode conditional healthiness, i.e. healthiness with regard to a particular health condition, but prior healthiness, i.e. healthiness of a food item per se. To further show that these two concepts are not the same, Table 9 displays the 20 food items that most strongly correlate with the conditional healthiness class (i.e. SUIT, $B E N E F$ and PREVENT) and the conditional unhealthiness class (i.e. UNSUIT, WORSEN, CAUSE). Correlation was determined with the help of Pointwise Mutual Information (Church and Hanks 1990). We denote the healthiness status according to the healthiness lexicon by + for healthy and - for unhealthy, respectively. While we observe some notable agreement between prior and conditional healthiness, there is some heavy disagreement between prior and conditional unhealthiness. Milchprodukte (dairy products), for example, are mostly considered healthy due to their nutrient content. However, for people with specific health conditions, e.g. Neurodermitis (dermatitis), they can actually be harmful. Similarly, Muscheln (mussels) and Pilze (mushrooms) are nutritious but for people with a sensitive stomach, they can cause nausea or bowel complaints.

Table 9 Top 20 food items that most strongly correlate with conditional healthiness and unhealthiness

\begin{tabular}{lll}
\hline Rank & Conditional Healthy & Conditional Unhealthy \\
\hline 1 & Hühnersuppe (chicken broth) + & Alkohol (alcohol) - \\
2 & Flohsamen (psyllium) + & Süßstoff (aspartame) - \\
3 & Zwiebelsaft (onion juice) + & Rhabarber (rhubarb) + \\
4 & Natron (baking soda) N/A & Kuhmilch (cow milk) + \\
5 & Salbei (salvia) + & Chips (crisps) - \\
6 & Salbeitee (salvia tea) + & Knoblauch (garlic) + \\
7 & Kräuter (herbs) + & Pilze (mushrooms) + \\
8 & Ingwertee (ginger tea) + & Schweinefleisch (pork) - \\
9 & Tee (tea) + & Milchprodukt (dairy product) + \\
10 & Thymiantee (thyme tea) + & Ei (egg) + \\
11 & Fenchelhonig (fennel honey) + & Fertiggericht (instant meal) - \\
12 & Ingwer (ginger) + & Geschmacksverstärker (flavour enhancer) - \\
13 & Saft (juice) + & Cocktail (cocktail) - \\
14 & Heilerde (healing earth) N/A & Spargel (asparagus) + \\
15 & Fenchel (fennel) + & Quark (curd cheese) + \\
16 & Suppe (soup) N/A & Muscheln (mussels) + \\
17 & Mineralwasser (mineral water) + & Weißmehl (white flour) - \\
18 & Paprika (bell pepper) + & Stevia (stevia) + \\
20 & Sirup (syrup) - & Roggen (rye) + \\
\hline & Radieschen (radish) + & Sekt (sparkling wine) - \\
\hline & &
\end{tabular}

+ and - denote the prior healthiness according to the healthiness lexicon (Wiegand et al. 2012c); bold type font denotes mismatch between prior and conditional healthiness 
Table 10 Description of the healthiness features

\begin{tabular}{|c|c|c|}
\hline Subgroup & Feature & Abbreviation \\
\hline \multirow[t]{4}{*}{ healthTarget } & Is target food item a priori healthy? & targetHlth \\
\hline & Is target food item a priori definitely healthy? & targetDefHlth \\
\hline & Is target food item a priori unhealthy? & targetUnh \\
\hline & Is target food item a priori definitely unhealthy? & targetDefUnh \\
\hline \multirow[t]{8}{*}{ healthCont } & Number of food items that are a priori healthy in target sentence only & hlthTS \\
\hline & $\begin{array}{l}\text { Number of food items that are a priori definitely healthy in target } \\
\text { sentence only }\end{array}$ & defHlthTS \\
\hline & $\begin{array}{l}\text { Number of food items that are a priori unhealthy in target sentence } \\
\text { only }\end{array}$ & unhTS \\
\hline & $\begin{array}{l}\text { Number of food items that are a priori definitely unhealthy in target } \\
\text { sentence only }\end{array}$ & defUnhTS \\
\hline & Number of food items that are a priori healthy in entire context & hlthEC \\
\hline & $\begin{array}{l}\text { Number of food items that are a priori definitely healthy in entire } \\
\text { context }\end{array}$ & defHlthEC \\
\hline & Number of food items that are a priori unhealthy in entire context & unhEC \\
\hline & $\begin{array}{l}\text { Number of food items that are a priori definitely unhealthy in entire } \\
\text { context }\end{array}$ & defUnhEC \\
\hline
\end{tabular}

All features are derived from the healthiness lexicon (Wiegand et al. 2012c); for the contextual features healthCont, the healthiness status of the target food item is not included

The specific features derived from that healthiness lexicon are listed in Table 10. They are divided into two groups. healthTarget describes the healthiness of the target food item. These features are designed to check in how far conditional healthiness correlates with the prior healthiness of the target food item. The second group of features, healthCont, encompasses the prior healthiness status of neighbouring food items in the given context of an instance. Thus, we want to find out in how far the prior healthiness knowledge about neighbouring food items helps to predict the conditional healthiness of the target food item. While there is definitely not a perfect correlation between prior and conditional healthiness when individual food items are considered (as shown in Table 9), it may still be the case that there is a stronger correlation between conditional healthiness and groups of healthy expressions co-occurring with each other rather than just a mention of a single healthy expression in a sentence. Moreover, for unknown target food items (i.e. food items that are not contained in the healthiness lexicon) these features might also be helpful (provided that there are mentions of known food items within the same instance) while the features from healthTarget could not make any prediction. Even though the healthiness lexicon is fairly large, it does not cover very exotic food items, such as Natron (baking soda) or Heilerde (healing earth), which are not unlikely to appear as target food items in our dataset. 


\subsection{Food and health condition priors}

We also want to examine in how far there is a correlation between health conditions and our class labels, on the one hand, and a correlation between food items and our class labels, on the other hand. The purpose of this investigation is twofold. Firstly, we use these correlations as a baseline. For example, if there is a strong correlation between certain health conditions and a particular class label irrespective of a particular target food item, we may wonder whether there is any point in a sophisticated textual analysis. (The same can, of course, be said if there is a strong correlation between certain food items and a particular class label.) In that case, one may equally well extract relations by just extracting food items frequently cooccurring with a particular health condition and then assigning the relation type with which the health condition mostly co-occurs. Secondly, we also want to examine whether the knowledge of these correlations can be usefully combined with the other information learnt from texts.

In order to further motivate feature engineering taking into consideration these types of correlations, we will have a look at the class distributions among the 10 most frequent health conditions and food items, as shown in Tables 11 and 12, respectively. With regard to the relation between health conditions and class labels (Table 11), we find that the class distribution varies quite a lot between the different conditions. In most cases, there is a tendency towards one group of classes, in other words, either the majority of instances belong to the subclasses of conditional healthiness (i.e. SUIT, BENEF and PREVENT) as it is most notably the case for Husten (cough) or Halsschmerzen (sore throat), or the majority of instances belong to the subclasses of conditional unhealthiness (i.e. UNSUIT, WORSEN and CAUSE), as it is the case for Neurodermitis (dermatitis). However, we also find that in many cases a significant amount of instances also belong to the other group of class labels.

Table 11 Distribution of the different classes among the 10 most frequent target health conditions

\begin{tabular}{|c|c|c|c|c|c|c|c|c|c|c|c|c|}
\hline \multirow[t]{3}{*}{ Condition } & \multicolumn{6}{|c|}{ Conditional Healthy } & \multicolumn{6}{|c|}{ Conditional Unhealthy } \\
\hline & \multicolumn{2}{|c|}{ SUIT } & \multicolumn{2}{|c|}{ BENEF } & \multicolumn{2}{|c|}{ PREVENT } & \multicolumn{2}{|c|}{ UNSUIT } & \multicolumn{2}{|c|}{ WORSEN } & \multicolumn{2}{|c|}{ CAUSE } \\
\hline & Freq & Perc & Freq & Perc & Freq & Perc & Freq & Perc & Freq & Perc & Freq & Perc \\
\hline Durchfall (diarrhoea) & 32 & 15.8 & 40 & 19.7 & 2 & 1.0 & 4 & 2.0 & 4 & 2.0 & 121 & 59.6 \\
\hline Sodbrennen (heartburn) & 26 & 14.2 & 39 & 21.3 & 54 & 2.2 & 10 & 5.5 & 1 & 0.6 & 103 & 56.3 \\
\hline Kopfschmerzen (headache) & 23 & 13.3 & 42 & 24.3 & 17 & 9.8 & 2 & 1.2 & 0 & 0.0 & 89 & 51.4 \\
\hline Husten (cough) & 34 & 23.5 & 93 & 61.1 & 2 & 1.4 & 4 & 2.8 & 5 & 3.5 & 7 & 4.8 \\
\hline Halsschmerzen (sore throat) & 53 & 42.4 & 67 & 53.6 & 0 & 0.0 & 3 & 2.4 & 1 & 0.8 & 1 & 0.1 \\
\hline Schwangerschaft (pregnancy) & 32 & 28.3 & 17 & 15.0 & 0 & 0.0 & 64 & 56.6 & 0 & 0.0 & 0 & 0.0 \\
\hline Diabetes (diabetes) & $\mathbf{5 0}$ & 56.2 & 7 & 7.9 & 1 & 1.1 & 26 & 29.2 & 1 & 1.1 & 4 & 4.5 \\
\hline Übelkeit (nausea) & 9 & 11.4 & 30 & 38.0 & 1 & 1.3 & 3 & 3.8 & 0 & 0.0 & 36 & 45.6 \\
\hline Neurodermitis (dermatitis) & 18 & 26.1 & 3 & 4.4 & 0 & 0.0 & 34 & 49.3 & 4 & 5.8 & 10 & 14.5 \\
\hline Fieber (fever) & 24 & 38.1 & 29 & 46.0 & 0 & 0.0 & 3 & 4.8 & 0 & 0.0 & 7 & 11.1 \\
\hline
\end{tabular}

Bold values indicate highest score in that row 
Table 12 Distribution of the different classes among the 10 most frequent target food items

\begin{tabular}{|c|c|c|c|c|c|c|c|c|c|c|c|c|}
\hline \multirow[t]{3}{*}{ Food item } & \multicolumn{6}{|c|}{ Conditional Healthy } & \multicolumn{6}{|c|}{ Conditional Unhealthy } \\
\hline & \multicolumn{2}{|c|}{ SUIT } & \multicolumn{2}{|c|}{ BENEF } & \multicolumn{2}{|c|}{ PREVENT } & \multicolumn{2}{|c|}{ UNSUIT } & \multicolumn{2}{|c|}{ WORSEN } & \multicolumn{2}{|c|}{ CAUSE } \\
\hline & Freq & Perc & Freq & Perc & Freq & Perc & Freq & Perc & Freq & Perc & Freq & Perc \\
\hline Tee (tea) & 46 & 48.9 & 36 & 38.3 & 3 & 3.2 & 2 & 2.1 & 0 & 0.0 & 7 & 7.5 \\
\hline Kaffee (coffee) & 12 & 16.9 & 19 & 26.8 & 10 & 14.1 & 11 & 15.5 & 2 & 2.8 & 17 & 23.9 \\
\hline Honig (honey) & 16 & 25.4 & 38 & 60.3 & 0 & 0.0 & 2 & 3.2 & 2 & 3.2 & 5 & 7.9 \\
\hline Alkohol (alcohol) & 0 & 0.0 & 1 & 2.0 & 0 & 0.0 & 29 & 59.2 & 0 & 0.0 & 19 & 38.8 \\
\hline Hühnersuppe (chicken broth) & 18 & 45.0 & 20 & 50.0 & 1 & 2.5 & 1 & 2.5 & 0 & 0.0 & 0 & 0.0 \\
\hline Fleisch (meat) & 7 & 18.0 & 6 & 15.4 & 7 & 18.0 & 11 & 28.2 & 1 & 2.6 & 7 & 18.0 \\
\hline Milk (milk) & 3 & 8.3 & 11 & 30.6 & 0 & 0.0 & 8 & 22.2 & 4 & 11.1 & 10 & 27.8 \\
\hline Cola (coke) & 15 & 41.7 & 9 & 25.0 & 1 & 2.8 & 2 & 5.6 & 3 & 8.3 & 6 & 16.7 \\
\hline Fett (fat) & 3 & 8.6 & 1 & 2.9 & 3 & 8.6 & 8 & 22.9 & 0 & 0.0 & 20 & 57.1 \\
\hline Wein (wine) & 5 & 15.6 & 2 & 6.3 & 0 & 0.0 & 1 & 3.1 & 0 & 0.0 & 24 & 75.0 \\
\hline
\end{tabular}

Bold values indicate highest score in that row

In the case of Übelkeit (nausea) there is almost a balance between instances belonging to the subclasses of conditional healthiness and unhealthiness. For a few health conditions, some of the class labels have not been observed at all. This mostly affects the class labels PREVENT and WORSEN. This can be ascribed to the general sparsity of these two class labels (Table 3). Beyond those two class labels, we only find that for Schwangerschaft (pregnancy) there is not a single instance labeled as CAUSE which is also absolutely plausible.

As far as the relation between food items and class labels are concerned (Table 12), we find similar results. On the one hand, there are food items which clearly display a tendency to appear with subclasses of conditional healthiness (e.g. Tee (tea), Honig (honey) or Hühnersuppe (chicken broth)) or subclasses of conditional unhealthiness (e.g. Alkohol (alcohol) or Fett (fat)), but there are also food items which are almost uniformly distributed throughout the different class labels (most notably Kaffee (coffee), Fleisch (meat) and Milch (milk)). It is also interesting to see that among some of the food items that have a tendency towards some specific class labels, these class labels need not be consistent with the respective prior healthiness of the food item (Sect. 3.5). This is most striking for the target food item Cola (coke) which strongly co-occurs with conditional healthiness, i.e. SUIT and BENEF. For example, it is often considered helpful for curing diarrhoea. However, Cola (coke) is generally considered to be unhealthy as far as its nutrient content is concerned. As a consequence, using the correlations between food items and our relation types based on observations from our corpus may be more effective for conditional healthiness classification than using a prior healthiness lexicon.

From this statistical analysis, we can conclude that there are tendencies of particular health conditions and food items to co-occur with particular classes or, more precisely, groups of classes. For supervised learning, we introduce two simple 
Table 13 Description of the prior features

\begin{tabular}{ll}
\hline Feature & Description \\
\hline condPrior & $\begin{array}{c}\text { Always assign an instance with a particular target health condition the relation type } \\
\text { with which it mostly co-occurs. }\end{array}$ \\
foodPrior & $\begin{array}{c}\text { Always assign an instance with a particular target food item the relation type with which } \\
\text { it mostly co-occurs. }\end{array}$ \\
\hline
\end{tabular}

features that exploit these correlations. condPrior always predicts for each instance in which a particular health condition occurs the class label with which it has mostly been observed on our dataset. For example, every instance of Durchfall (diarrhoea) is classified as CAUSE (Table 11). Similarly, foodPrior always predicts for each instance in which a particular food item occurs the class label with which it mostly co-occurs. For example, every instance of Tee (tea) is classified as SUIT (Table 12). Table 13 summarizes the two prior features we use in this article.

We already stated in Sect. 2.1 that while the health conditions chosen on our dataset are, by and large, frequently occurring expressions, there were no restrictions made on the food items we consider. As a consequence, the occurrences of food items will follow a power law distribution (Zipf's law), that is, there are many infrequent food items, i.e. $40 \%$ of them are singletons. Further evidence regarding the different distributions of health conditions and food items can be obtained by considering the average number of instances associated with a particular item: The average number of instances associated with a health condition is 64 while it is only 7 for food items. For rare food items, in particular singletons, it does not make sense to determine the most frequently observed class label, as these observations are not very reliable. (They could happen by chance.) Another negative side-effect of including them for our feature foodPrior would be that they overfit. For instance, foodPrior would predict the correct label for every singleton. In order to measure a more realistic impact of this feature, we restrain the prediction to only those food items which occur at least 10 times on our dataset. This also means that for almost $30 \%$ of the instances no prediction can be made by foodPrior as these instances contain target food items that are too rare.

\subsection{Manually chosen keywords}

In various text classification tasks, the performance of classifiers can usually be improved by using task-specific lexicons that comprise words that are predictive for the classes that are to be detected. One usually applies such lexicons for features that ask if (or how many) of those predictive words occur in a text passage that is to be classified. Such features are usually less sparse than plain bag-of-words features since they generalize over individual words and may also make predictions for words that have not been observed in the training data (provided the words are contained in the respective lexicons). 
Table 14 Features derived from keyword lexicons

\begin{tabular}{ll}
\hline Subgroup & Feature \\
\hline $\mathrm{KW}$ & Number of keywords of relation type SUIT \\
& Number of keywords of relation type BENEF \\
& Number of keywords of relation type PREVENT \\
& Number of keywords of relation type UNSUIT \\
& Number of keywords of relation type WORSEN \\
& Number of keywords of relation type CAUSE \\
& Is target food item syntactically related to keyword of relation type SUIT? \\
& Is target food item syntactically related to keyword of relation type BENEF? \\
SyntKW & Is target food item syntactically related to keyword of relation type PREVENT? \\
& Is target food item syntactically related to keyword of relation type UNSUIT? \\
& Is target food item syntactically related to keyword of relation type WORSEN? \\
& Is target food item syntactically related to keyword of relation type CAUSE? \\
& Is target health condition syntactically related to keyword of relation type SUIT? \\
& Is target health condition syntactically related to keyword of relation type BENEF? \\
Is target health condition syntactically related to keyword of relation type PREVENT? & Is target health condition syntactically related to keyword of relation type $U N S U I T ?$ \\
Is target health condition syntactically related to keyword of relation type WORSEN? \\
Is target health condition syntactically related to keyword of relation type CAUSE?
\end{tabular}

For the set of task-specific linguistic features (Sect. 3.2), we already included such type of features, i.e. causeTS and causeEC (Table 6). Since in this article, we present a fairly novel task with new class labels, with the exception of the class CAUSE, there do not exist any appropriate lexicons tailored to these classes that we could use. As a consequence, it is difficult to create such word lists in an unbiased manner. The only way to create them is with the help of our dataset. However, this may result in overfitting those lexicons to the data on which we test our classifiers. Despite this risk, we manually create such lists in that fashion. However, being aware of the fact that the result may be biased, we regard the corresponding features as an upper bound for lexical features in general.

For each relation type, we consider two types of features (Table 14). $K W$ contains plain features that consider the keywords for the pertaining relation type in the target sentence. In addition, Synt $K W$ includes for each relation type a feature that asks whether the target sentence contains at least one keyword (for the pertaining relation type) that is either syntactically related to the target food item or the target health condition. SyntKW is analogous to polarSynt (Table 8) and has also been included with a similar motivation (Sect. 3.4).

In order to obtain the keywords, in a first step, each noun, verb or adjective in a target sentence considered to be predictive towards the label that the corresponding instance was assigned was marked. We only annotated unigrams. In a second step, all expressions that had thus been marked were collected, and for feature extraction, 
Table 15 The 10 most frequent keywords for each class

\begin{tabular}{|c|c|c|c|c|c|}
\hline SUIT & BENEF & PREVENT & UNSUIT & WORSEN & CAUSE \\
\hline $\begin{array}{l}\text { gut } \\
\text { (good) }\end{array}$ & $\begin{array}{l}\text { helfen } \\
\text { (help) }\end{array}$ & $\begin{array}{l}\text { vorbeugen } \\
\text { (prevent) }\end{array}$ & $\begin{array}{l}\text { weglassen } \\
\text { (leave out) }\end{array}$ & $\begin{array}{l}\text { fördern } \\
\text { (increase) }\end{array}$ & $\begin{array}{l}\text { bekommen } \\
\text { (get) }\end{array}$ \\
\hline $\begin{array}{l}\text { können } \\
\text { (be allowed to) }\end{array}$ & $\begin{array}{l}\text { wirken } \\
\text { (be effective) }\end{array}$ & $\begin{array}{l}\text { vermeidbar } \\
\text { (avoidable) }\end{array}$ & $\begin{array}{l}\text { verzichten } \\
\text { (do without) }\end{array}$ & $\begin{array}{l}\text { verschleimen } \\
\text { (congest) }\end{array}$ & $\begin{array}{l}\text { verursachen } \\
\text { (cause } v \text { ) }\end{array}$ \\
\hline $\begin{array}{l}\text { empfehlen } \\
\text { (recommend) }\end{array}$ & $\begin{array}{l}\text { Mittel } \\
\text { (remedy) }\end{array}$ & $\begin{array}{l}\text { vermeiden } \\
\text { (avoid) }\end{array}$ & $\begin{array}{l}\text { meiden } \\
\text { (avoid) }\end{array}$ & $\begin{array}{l}\text { verschlimmern } \\
\text { (worsen) }\end{array}$ & $\begin{array}{l}\text { führen (zu) } \\
\text { (lead (to)) }\end{array}$ \\
\hline $\begin{array}{l}\text { dürfen } \\
\text { (may) }\end{array}$ & $\begin{array}{l}\text { Hausmittel } \\
\text { (home remedy) }\end{array}$ & $\begin{array}{l}\text { schützen } \\
\text { (protect) }\end{array}$ & $\begin{array}{l}\text { tabu } \\
\text { (taboo) }\end{array}$ & $\begin{array}{l}\text { reizen } \\
\text { (irritate) }\end{array}$ & $\begin{array}{l}\text { kriegen } \\
\text { (catch) }\end{array}$ \\
\hline $\begin{array}{l}\text { vertragen } \\
\text { (agree with) }\end{array}$ & $\begin{array}{l}\text { lindern } \\
\text { (abate) }\end{array}$ & $\begin{array}{l}\text { protektiv } \\
\text { (protective) }\end{array}$ & $\begin{array}{l}\text { Problem } \\
\text { (problem) }\end{array}$ & $\begin{array}{l}\text { umhauen } \\
\text { (knock out) }\end{array}$ & $\begin{array}{l}\text { reagieren } \\
\text { (react) }\end{array}$ \\
\hline $\begin{array}{l}\text { umstellen } \\
\text { (switch) }\end{array}$ & $\begin{array}{l}\text { bekämpfen } \\
\text { (combat) }\end{array}$ & $\begin{array}{l}\text { herzschützend } \\
\text { (heart-protecting) }\end{array}$ & $\begin{array}{l}\text { einschränken } \\
\text { (cut back) }\end{array}$ & $\begin{array}{l}\text { stark } \\
\text { (strong) }\end{array}$ & $\begin{array}{l}\text { kommen (von) } \\
\text { (come (from)) }\end{array}$ \\
\hline $\begin{array}{l}\text { schwören } \\
\text { (swear by) }\end{array}$ & $\begin{array}{l}\text { lösen } \\
\text { (expectorate) }\end{array}$ & $\begin{array}{l}\text { ersparen } \\
\text { (spare) }\end{array}$ & $\begin{array}{l}\text { Verzicht } \\
\text { (waiver) }\end{array}$ & $\begin{array}{l}\text { schüren } \\
\text { (stir up) }\end{array}$ & $\begin{array}{l}\text { auslösen } \\
\text { (trigger) }\end{array}$ \\
\hline $\begin{array}{l}\text { rutschen } \\
\text { (slide down) }\end{array}$ & $\begin{array}{l}\text { Heilmittel } \\
\text { (cure) }\end{array}$ & $\begin{array}{l}\text { abwehren } \\
\text { (combat) }\end{array}$ & $\begin{array}{l}\text { vermeiden } \\
\text { (avoid) }\end{array}$ & $\begin{array}{l}\text { schleimbildend } \\
\text { (mucous) }\end{array}$ & $\begin{array}{l}\text { machen } \\
\text { (create) }\end{array}$ \\
\hline $\begin{array}{l}\text { (es) geht } \\
\text { (okay) }\end{array}$ & $\begin{array}{l}\text { Medizin } \\
\text { (medicine) }\end{array}$ & $\mathrm{N} / \mathrm{A}^{\mathrm{a}}$ & $\begin{array}{l}\text { schlecht } \\
\text { (bad) }\end{array}$ & $\begin{array}{l}\text { ruinieren } \\
\text { (ruin) }\end{array}$ & $\begin{array}{l}\text { hervorrufen } \\
\text { (give rise to) }\end{array}$ \\
\hline $\begin{array}{l}\text { geeignet } \\
\text { (suitable) }\end{array}$ & $\begin{array}{l}\text { vertreiben } \\
\text { (combat) }\end{array}$ & $\mathrm{N} / \mathrm{A}^{\mathrm{a}}$ & $\begin{array}{l}\text { schädlich } \\
\text { (harmful) }\end{array}$ & $\begin{array}{l}\text { gefährlich } \\
\text { (dangerous) }\end{array}$ & $\begin{array}{l}\text { Ursache } \\
\text { (cause } n \text { ) }\end{array}$ \\
\hline
\end{tabular}

${ }^{a}$ For PREVENT only 8 keywords were manually extracted

Table 16 Proportion of instances with at least one keyword (in target sentence)

\begin{tabular}{llllll}
\hline SUIT & BENEF & PREVENT & UNSUIT & WORSEN & CAUSE \\
\hline 21.73 & 56.97 & 13.51 & 32.39 & 73.33 & 72.20 \\
\hline
\end{tabular}

any mention of them is considered a keyword. For illustration, Table 15 lists the 10 most frequent keywords for each relation type on our dataset.

Unfortunately, not in all instances such keywords could be identified. Table 16 shows the proportion of instances for which at least one keyword was identified. The categories for which the fewest keywords could be identified are SUIT, PREVENT and UNSUIT. Typical sentences for these categories (in which no keywords could be marked) are Sentences (22)-(24). These examples neither contain keywords with parts of speech other than the ones we consider nor keywords being multiword expressions. In all cases, the relations are inferred. In Sentence (22), the fact that the speaker has Blasentee (diuretic tea) in her cupboard is the consequence of regularly suffering from cystitis. One, therefore, concludes that Blasentee (diuretic tea) is a suitable food item for this health condition. (We refrained from labeling this 
instance as $B E N E F$ as there is no explicit mention that the consumption of that tea actually cures the illness.) In Sentence (23), one infers that the reason why the Eskimo did not suffer from heart diseases is that they mostly ate fish. There is no lexical cue explicitly indicating that causal relationship. ${ }^{10}$ Sentence (24) is a rhetoric question. This figure of speech clearly implies some disapproval on the part of the speaker regarding the behaviour of the addressee, that is, the speaker thinks that the consumption of Marmelade (jam) is unsuitable for people suffering from diabetes.

(22) Ich habe immer Blasentee im Schrank (habe so etwa 3-4 mal jährlich eine Blasenentzündung).

(I always have some diuretic tea in my cupboard (usually get cystitis two to three times a year).) LABEL: SUIT

(23) Und die Eskimos lebten früher überwiegend von Fisch, sie kannten keine Herzkrankheiten.

(And in the past, the Eskimo predominantly consumed fish; they did not have any heart diseases.) LABEL: PREVENT

(24) Du hast Diabetes und kochst tonnenweise Marmelade ein?

(You suffer from diabetes and you are canning tons of jam?) LABEL: UNSUIT

Instances like Sentences (22)-(24) cannot be modelled reliably by state-of-the-art computational models. By employing the keyword features, we may obtain an estimate of how good we would be able to detect the different relation types with an ideal list of keywords but not being able to deal with inferred relations (such as Sentences (22)-(24)).

\section{Experiments}

In this section, we describe our experiments to detect the different classes that describe some relationship between target food item and health condition. We only focus on the prediction of the four relation types SUIT (Sect. 2.3.1), BENEF (Sect. 2.3.2), UNSUIT (Sect. 2.3.4) and CAUSE (Sect. 2.3.6). (Instances of the other relation types remain in our dataset and should be considered exclusively as negative data instances. $)^{11} \mathrm{We}$ do not address the classification of the two negative classes EMBREL (Sect. 2.3.7) and NOREL (Sect. 2.3.8) since people are not interested in sentences in which a food item and a health condition occur but there is no relationship between them (NOREL), or there is a relationship but this is embedded in a context so that one cannot conclude that the relation type holds (EMBREL). The remaining two relation types PREVENT (Sect. 2.3.3) and WORSEN (Sect. 2.3.5) are not considered since they are too infrequent (Table 3); neither of these relation types covers more than $3 \%$ of the instances. Our initial experiments with these types showed that this size does not suffice for supervised

\footnotetext{
10 The fact that the target health condition is negated may indicate that this sentence expresses some type of conditional unhealthiness, however, there is no lexical cue that helps us to identify the subtype PREVENT.

11 By negative (data) instances, we mean those instances that have not been tagged with the relation type that is to be extracted.
} 
classification. We assume that the sparseness of those two relation types is not an artifact of our dataset but reflects their general distribution. Therefore, ignoring them does not mean overlooking crucial relation types. Presumably, the relation type PREVENT is rare since food items are a less reliable means to prevent illnesses from breaking out than, for instance, vaccinations. WORSEN is rare since it is actually a variation of UNSUIT in which the deterioration is explicitly mentioned. Our comparison of instances of UNSUIT and WORSEN actually revealed that the food items that were mentioned for a particular health condition do not vary between these two relation types. Therefore, we would recommend subsuming instances of WORSEN by UNSUIT in future work. ${ }^{12}$

Each instance to be classified is a sentence in which there is a co-occurrence of a target food item and a health condition along its respective context sentences (Sect. 2.2). The dataset was parsed using the Stanford Parser (Rafferty and Manning 2008). We carry out a 5-fold cross-validation on our manually labeled dataset. For the supervised classifier, we chose Support Vector Machines (Joachims 1999). As a toolkit, we use SVMLight ${ }^{13}$ with a linear kernel.

\subsection{Comparison of task-specific features}

In our first experiment we look at various task-specific features. By that we mean the task-specific linguistic features (Sect. 3.2), the sentiment features (Sect. 3.4) and the features derived from a healthiness lexicon (Sect. 3.5).

We contrast them with two baseline features being coocc and the word-based features word (Sect. 3.1). coocc is an unsupervised classifier that considers all instances of our dataset as positive instances (of the class which is examined, i.e. SUIT, BENEF, UNSUIT or CAUSE). In other words, this baseline indicates how well the mere co-occurrence of the target food item and the target health condition predicts any of our four classes. ${ }^{14}$

\subsubsection{Comparison of feature groups}

We first compare the performance of different feature groups. Table 17 shows the results of this comparison for each class. If we use either the sentiment features (i.e. polarWord and polarSynt) or the features derived from the healthiness lexicon (i.e. healthTarget and healthCont) in isolation, for many classes the resulting performance remains as the simple baseline coocc. For the healthiness features, this may come as a surprise, particularly if one recalls that there is some correspondence between conditional healthiness and the prior healthiness as shown in Table 9. One should recall, however, that our dataset does not exclusively consist of sentences in

\footnotetext{
12 Since the instances labeled as WORSEN only cover approximately $1 \%$ of our entire dataset (Table 3), we are convinced that the choice of treating them as negative instances or as instances of type UNSUIT will not affect the overall results of our experiments.

13 http://svmlight.joachims.org.

${ }^{14}$ We also experimented with variations restricting the co-occurrence to a fixed window size. However, we did not obtain better classifiers than with the plain (sentence-wise) co-occurrence.
} 


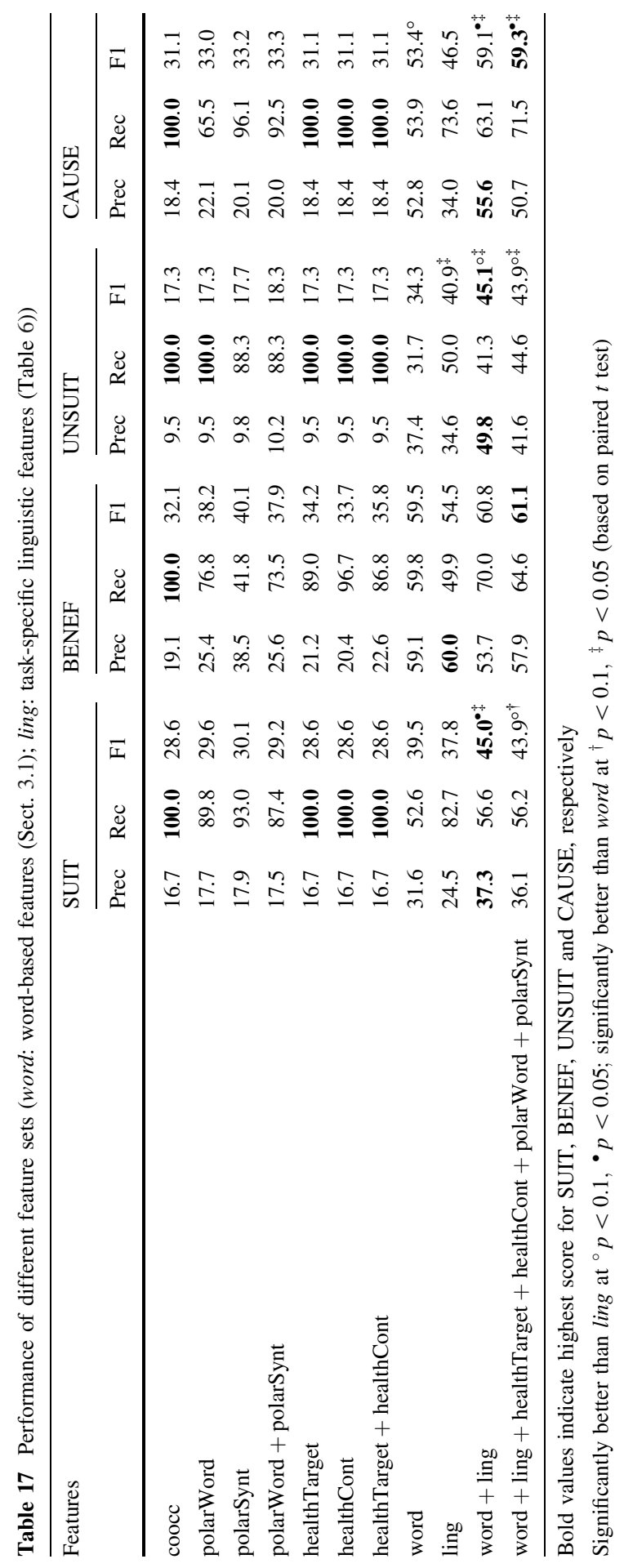


which the target food item is claimed to be suitable or unsuitable given a particular health condition (in that case, the scores produced by the healthiness features would presumably have been higher). There is also a large number of sentences in which the cooccurrence of the target food item and health condition does neither express suitability nor unsuitability, that is, these are instances of the types NOREL or EMBREL (Table 3). For the exclusion of those cases, the health features are totally ineffective.

Table 17 also shows that considering four fine-grained classes (i.e. SUIT, BENEF, UNSUIT and CAUSE) instead of two coarse-grained (i.e. suitability and unsuitability) is appropriate, since different features are effective for the different fine-grained classes. This mostly concerns the two relation types SUIT and BENEF (which would be merged to the class suitability). As far as BENEF is concerned, both the sentiment and the healthiness features beat the baseline feature $\operatorname{coocc}$. We have following explanations: If a food item is classified as $B E N E F$, the food item has some curing properties. In order to express this property, one has to use polar expressions. In addition, having some curing function also usually coincides with healthiness. The situation is different for SUIT which to our definition in Sect. 2.3.1 means that one does not expect a negative effect on the health of an afflicted person. In terms of verbalizing such weaker property, we assume less usage of polar expressions. In addition, a food item that is not harmful is less likely to be healthy (in general) than a food item with a curing function. In Table 16, we found that the proportion of keywords within the set of instances labeled as $B E N E F$ is much larger than the proportion within instances labeled as SUIT. This is consistent with our observation that for $B E N E F$ polar expressions have a notable impact on the classification performance as polar expressions are some kind of keywords.

As far as UNSUIT and CAUSE are concerned, the healthiness features do not work at all. In Table 9, we already found that conditional unhealthiness correlates with prior unhealthiness much less than conditional healthiness does with prior healthiness. The sentiment features only have a marginal positive effect on UNSUIT and CAUSE which means that negative polar expressions are not that indicative of conditional unhealthiness, either.

The simple word-based features word are much more effective than the sentiment and healthiness features. On all relation types coocc is beaten by a large margin. The same can be said about our task-specific features ling. In addition, the combination of the two types of features also results in some improvement. Adding the healthiness and sentiment features, however, has no notable effect. From these results, we conclude that a contextual analysis (as enabled by the features word and ling) is more effective than focusing on external knowledge (as the healthiness and sentiment features do) in this classification task.

\subsubsection{Comparison of individual features}

We now turn to the performance of individual features. Table 18 shows the most highly ranked task-specific features for the four different classes according to Chisquare ranking. The feature ranking was carried out with WEKA (Witten and Frank 2005). We consider the individual features from the three different high-level feature types, that is, sentiment features, healthiness features and linguistic features. 
Table 18 Top 15 task-specific features according to Chi-square feature ranking for each individual class

\begin{tabular}{|c|c|c|c|c|c|c|c|}
\hline \multicolumn{2}{|l|}{ SUIT } & \multicolumn{2}{|l|}{ BENEF } & \multicolumn{2}{|l|}{ UNSUIT } & \multicolumn{2}{|l|}{ CAUSE } \\
\hline Feature & Score & Feature & Score & Feature & Score & Feature & Score \\
\hline synoHlthTS $^{\mathrm{a}}$ & 46.1 & synoHlthTS $^{\mathrm{a}}$ & 315.3 & negFood $^{\mathrm{a}}$ & 262.3 & causeTS $^{\mathrm{a}}$ & 172.6 \\
\hline foodBefCond $^{\mathrm{a}}$ & 43.5 & againstCond $^{\mathrm{a}}$ & 310.7 & synoHlthEC ${ }^{a}$ & 18.8 & foodBefCond $^{\mathrm{a}}$ & 141.1 \\
\hline againstCond $^{\mathrm{a}}$ & 31.4 & synoHlthEC ${ }^{\mathrm{a}}$ & 253.8 & againstCond $^{\mathrm{a}}$ & 18.0 & causeEC $^{\mathrm{a}}$ & 114.1 \\
\hline negFood $^{\mathrm{a}}$ & 25.5 & sPosCondSynt ${ }^{\mathrm{b}}$ & 238.5 & sPosCondSynt ${ }^{\mathrm{b}}$ & 15.7 & targetDefUnh ${ }^{\mathrm{c}}$ & 62.9 \\
\hline causeTS $^{\mathrm{a}}$ & 21.5 & posCondSynt $^{b}$ & 212.3 & causeEC $^{\text {a }}$ & 14.8 & negTS ${ }^{b}$ & 62.4 \\
\hline posCondSynt ${ }^{\mathrm{b}}$ & 20.5 & sPosTS $^{b}$ & 93.4 & foodBefCond $^{\mathrm{a}}$ & 14.5 & synoHlthEC ${ }^{\mathrm{a}}$ & 60.2 \\
\hline sPosCondSynt $^{\mathrm{b}}$ & 19.4 & sNegTS $^{b}$ & 90.9 & synoUnhEC ${ }^{a}$ & 13.6 & $n e g E C^{b}$ & 53.9 \\
\hline sPosTS $^{b}$ & 18.1 & sPosFoodSynt $^{\mathrm{b}}$ & 90.4 & targetUnh ${ }^{\mathrm{c}}$ & 11.8 & synoHlthTS $^{\mathrm{a}}$ & 51.6 \\
\hline $\operatorname{posTS}^{b}$ & 14.8 & negFood $^{\mathrm{a}}$ & 60.9 & synoHlthTS $^{\mathrm{a}}$ & 11.1 & targetUnh ${ }^{\mathrm{c}}$ & 50.9 \\
\hline synoHlthEC ${ }^{a}$ & 14.0 & sPosEC $^{\mathrm{b}}$ & 47.4 & targetDefUnh ${ }^{\mathrm{c}}$ & 10.9 & targetHlth ${ }^{\mathrm{c}}$ & 41.8 \\
\hline causeEC $^{\text {a }}$ & 12.8 & posFoodSynt $^{\mathrm{b}}$ & 44.7 & $\operatorname{defUnhTS}{ }^{c}$ & 10.5 & $\operatorname{posEC}^{b}$ & 41.7 \\
\hline $\operatorname{targetUnh}{ }^{\mathrm{c}}$ & 10.6 & $\mathrm{sNegEC}^{\mathrm{b}}$ & 36.2 & causeTS $^{\mathrm{a}}$ & 10.5 & againstCond $^{\mathrm{a}}$ & 38.6 \\
\hline sNegTS $^{b}$ & 10.5 & diseaseEC $^{\mathrm{a}}$ & 31.7 & sNegEC $^{b}$ & 9.9 & posCondSynt ${ }^{\mathrm{b}}$ & 35.2 \\
\hline negCond ${ }^{\mathrm{a}}$ & 9.7 & $\operatorname{targetDefUnh}^{\mathrm{c}}$ & 29.6 & sPosEC $^{b}$ & 8.9 & negFoodSynt $^{\mathrm{b}}$ & 34.5 \\
\hline irrealis $^{\mathrm{a}}$ & 9.5 & $\operatorname{posTS}^{\mathrm{b}}$ & 28.8 & defUnhEC ${ }^{c}$ & 7.7 & sPosFoodSynt ${ }^{b}$ & 33.9 \\
\hline
\end{tabular}

\footnotetext{
${ }^{a}$ Linguistic features (Table 6)

${ }^{\mathrm{b}}$ Sentiment features (Table 8)

${ }^{c}$ Features derived from healthiness lexicon (Table 10)
}

Even though we find features from all feature groups on those rankings, the top 3 ranks are always linguistic features. The healthiness features are pretty rare on SUIT and $B E N E F$. Among those few that occur are the features that describe the healthiness of the target rather than the healthiness of contextual food items. This implies that the healthiness status of neighbouring food items is less relevant. The sentiment features are exceptionally frequent on the highest ranks of BENEF. This is consistent with Table 17. The strongest sentiment features are those that check whether there is a syntactic relationship between some polar expression and the target food item or health condition.

In terms of absolute score of the individual features, we find that even the most highly ranked features of SUIT are much weaker than their counterparts of the other relation types. For $B E N E F$, there are five features with an exceptionally high score: Two features look for synonyms of the word healthy, i.e. synoHlth(EC|TS), one additional feature checks whether the health condition is preceded by against, i.e. againstCond, and two further sentiment features check whether the target health condition is syntactically related to some positive polar expression, i.e. sPosCondSynt and posCondSynt. The only feature with an exceptionally high correlation score for UNSUIT is the feature checking whether the food item is negated, i.e. negFood. If we revisit the most frequent keywords for that class (Table 15) we find that many of these words (e.g. weglassen (leave out), verzichten 
Table 19 Comparison of order between food item and health condition

\begin{tabular}{ll}
\hline Instances & Percentage of food items preceding health condition \\
\hline all instances & 55.42 \\
instances (manually) labeled as CAUSE & 78.84 \\
\hline
\end{tabular}

(do without), vermeiden (avoid) and tabu (taboo)) convey a request to stop consuming the target food item. These are shifters (Wilson et al. 2005), i.e. (predominantly) verbs, nouns and adjectives that express some negation but without being traditional negation expressions. Due to the semantic similarity between shifters and negation words, we can conclude that the strong predictiveness of negFood is consistent with these shifter keywords. (We will also discuss the importance of negation in our error analysis in Sect. 5.6.) The fact that the causekeywords from Girju et al. (2003), i.e. cause(EC|TS), are exceptionally predictive for CAUSE comes as no surprise and proves that this domain-independent list of cues is also predictive for our task.

We find it interesting that another exceptionally strong feature for the relation type CAUSE checks whether the target food item precedes the health condition in the target sentence, i.e. foodBefCond. Apparently, the temporal order of events that underlie a causation relation, i.e. the consumption of some food item followed by the outbreak of a disease, is reflected in the order in which these events are mentioned in a written text. To further substantiate this hypothesis, we also measured the proportion of cases in which the target food item preceded the health condition on all instances and on instances labeled as CAUSE. The result is displayed in Table 19. It indeed shows that while, in general, there is no notable tendency for a particular order (55.4\%), in $78.8 \%$ of the cases labeled as CAUSE, however, the food item precedes the health condition.

In Sect. 3.4, we pointed out the similarity between the sentiment features and the task-specific linguistic features $\operatorname{synoHlth}(E C \mid T S)$ and $\operatorname{synoUnh}(E C \mid T S)$. Table 18 gives evidence that the small set of these domain-specific expressions is more effective than the sentiment features (synoHlthTS is the strongest feature for SUIT and BENEF while synoHlthEC is the second strongest feature for UNSUIT).

On all feature rankings, we observe both features that consider the entire context (suffix-EC) and features that exclusively consider the target sentence (suffix-TS). This means that it is inconclusive which contextual scope is most informative for this task.

Table 20 shows the best performing feature subset for each relation type using a best-first forward selection. For this feature selection, we, again, use WEKA. This form of feature selection is complementary to that depicted in Table 18 as it excludes redundant features. It supports our previous observation that the sentiment and healthiness features are much less important than the task-specific linguistic features. No relation type has more than one sentiment and healthiness feature (each) on the list. The best performing feature set for UNSUIT even exclusively comprises linguistic features. Apart from that, some features seem to be much more important than their Chi-square ranking position suggests. This is especially true for 
irrealis, which appears on three of the four lists. Although its individual predictiveness towards the different classes is marginal (Table 18), the information it encodes is unique and cannot be obtained by other existing features (Table 20).

\subsection{Impact of generic linguistic features}

In this section, we examine the generic linguistic features. In the previous section, we found that the task-specific linguistic features systematically have a positive impact on classification performance. We want to know whether this contribution can be equally achieved by more generic (and thus simpler) features and/or whether the generic features can be usefully combined with the task-specific features. As a baseline feature set we use the word-based features to which the other features are added. Table 21 shows the result of this comparison.

The table shows that the syntactic relation path from the target food item to the target health condition, i.e. path, is less helpful than the part-of-speech features, i.e. pos. For three out of the four relation types (i.e. SUIT, UNSUIT and CAUSE), pos increases performance when added to word. path only manages improvements for the relation types SUIT and CAUSE. A combination of the two features does not result in a systematic improvement. The word-based features combined with the part-of-speech information are not as good as the word-based features combined with the task-specific linguistic features (ling). This means that the task-specific features cannot be replaced by the generic features. However, the combination of these features, i.e. pos and ling, results in some further improvement for the relation types $B E N E F$ and CAUSE.

\subsection{Impact of food and health condition priors}

In this section, we investigate the impact of the food and health condition priors (Table 13). Each of the priors is evaluated as a stand-alone feature and in combination with the best subset of features we obtained in our previous experiments, i.e. word + pos + ling. Table 22 displays the results.

The table shows that the priors themselves often largely outperform the standard baseline coocc. The most effective prior uses the knowledge about the target health condition, i.e. condPrior. However, the performance of the feature set word + pos + ling is significantly better in three out of four cases (UNSUIT is the only exception). This means that beyond this prior knowledge there is much more information that can be learnt from labeled textual data. As far as UNSUIT is concerned, there is a high fluctuation among the different folds (in cross-validation) so that the overall improvement of the feature set word + pos + ling is not statistically significant. Nevertheless, the classifiers based on that feature set have (overall) a much higher precision than the classifiers based on priors (which can be preferable for certain practical applications). The fact that we also gain a notable performance increase by combining the prior features with the feature set word + pos + ling for the relation types SUIT and CAUSE means that these two types of information are complementary to a certain extent. 


\subsection{Impact of manually chosen keywords}

Table 23 compares the best (overall) feature set from our previous experiments, i.e. word + pos + ling + condPrior + foodPrior from Table 22 (we denote it as no KW in Table 23) with manually extracted keywords. Even though we do not address the automatic extraction of instances of the relation types PREVENT and WORSEN, we included keyword features for these relation types (Table 14). Thus, the classifier may use them as negative features for the detection of the four remaining relation types (i.e. SUIT, BENEF, UNSUIT and CAUSE).

Overall, the inclusion of such keywords results in some increase in F-score. Adding syntactic information (SyntKW) has no notable impact on performance. Given the contribution of other features we explored in our previous experiments and considering that all features based on manually extracted keywords may also be biased towards our dataset, their impact is, in general, comparatively moderate. Thus, we conclude that extracting keywords for the different relation types is hardly one of the most promising directions for further improvement.

\section{Error analysis}

In this section, we present a detailed error analysis. It mostly focuses on explaining why certain features did not show the expected positive effect in our experiments. We address healthiness features (Sect. 5.1), linguistic features (Sect. 5.2) and sentiment features (Sect. 5.3). We also try to uncover why bag of words performs so well (Sect. 5.4) and also compare it with syntactic features (Sect. 5.5). Regarding the different relation types, we have a closer look at SUIT and UNSUIT (Sect. 5.6), which systematically scored lower than the remaining relation types. Since we use a dataset originating from user-generated web documents, we also need to address the general text quality of our corpus (Sect. 5.7). Finally, in Sect. 5.8, we merge the four fine-grained classes to two coarse-grained classes in order to check whether low classification scores are the result of an inventory of classes that are not sufficiently well-defined.

\subsection{Healthiness features}

In our evaluation, none of the features derived from the healthiness lexicon (Sect. 3.5) helped to produce better results than the bag of words and the taskspecific features (Sect. 3.2), i.e. word and ling in Table 17. Basically, this result should not be considered as an issue for error analysis. It just means that prior healthiness as encoded in the healthiness features (Table 10) does not sufficiently correlate with conditional healthiness. This should not come as a surprise if we recall the results of Table 9 displaying the food items strongly correlating with conditional healthiness. It was found that many food items generally considered healthy (e.g. Pilze (mushrooms) or Spargel (asparagus)) are often considered problematic for certain types of health conditions-so they are conditional unhealthy. As a matter of fact this insight should be regarded as some further 
motivation to consider conditional healthiness as a separate task which requires a different kind of feature engineering than prior healthiness. (Otherwise, if there were a very strong correlation between conditional and prior healthiness, we could solve the current task by re-using the methods from solving prior healthiness classification.)

\subsection{Linguistic features}

In our previous experiments, we could establish that, as a whole, our set of taskspecific features both improves a set of standard features (i.e. word + path + pos in Table 21) and that some individual features are especially predictive (e.g. againstCond for BENEF, negFood for UNSUIT or foodBefCon for CAUSE (Table 18)). However, among the set of those task-specific features, there are also features that neither appear to be predictive (Table 18) nor provide complementary information to the remaining features (Table 20). The features that fall within this category are question, side, scope and weird.

Table 24 lists the frequency of those less effective features in our dataset. For comparison, we also list the frequency of some effective features. If features are too rare, they are very unlikely to improve classification performance. Table 24 shows, however, that the less effective features are not less frequent than the effective features. From that we conclude that the sparsity of those features is not the reason for them to perform poorly.

Table 25 shows which other features (i.e. effective features) strongly correlate with those poor performing features. If there are strongly correlating features, this explains why these four less effective features were not listed on Table 20 (that only lists features that provide complementary information). Furthermore, if there are features overlapping with those less effective features, the correlating features should then be considered a better alternative. The fewer overlapping features there are, the more unique the information is of a particular feature. We find that the features negFoodSynt, boundary and sNegFoodSynt are the three features most strongly correlating with scope, having a Chi-square score of 98.9, 85.4 and 60.9, respectively. What all these features have in common is that they syntactically restrict the mention of the food item in a sentence: It comes as no surprise that boundary and scope correlate, as we introduced the former as a back-off feature of the latter (Table 6). ${ }^{15}$ The other two features, i.e. negFoodSynt and sNegFoodSynt, do not restrict target food item and health condition to be in the same clause (as scope does) but demand target food item and negative polar expression to be syntactically related. The feature scope can be considered a pre-requisite of the features negFoodSynt and sNegFoodSynt, so there is some linguistic connection between these features. The strong correlations between question and boundary as well as between weird and foodBefCond may not appear intuitive. However, one has to recall that two statistically correlated features do not have to be conceptually

\footnotetext{
${ }^{15}$ In Sect. 3.2 we already speculated that the performance of scope may be affected by a bad parse quality due to the noise contained in our language data. Obviously, the noise really affects syntactic processing and some of the features that depend on that information, such as scope.
} 


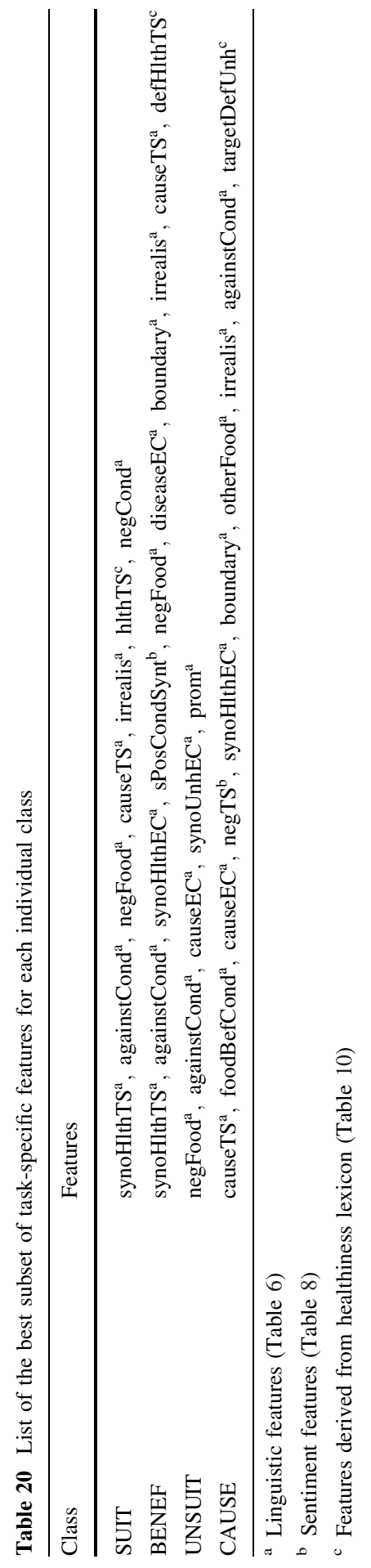


Table 21 Performance of generic linguistic features (Table 7)

\begin{tabular}{|c|c|c|c|c|c|c|c|c|c|c|c|c|}
\hline \multirow[t]{2}{*}{ Features } & \multicolumn{3}{|c|}{ SUIT } & \multicolumn{3}{|c|}{ BENEF } & \multicolumn{3}{|c|}{ UNSUIT } & \multicolumn{3}{|c|}{ CAUSE } \\
\hline & Prec & Rec & $\mathrm{F} 1$ & Prec & $\operatorname{Rec}$ & $\mathrm{F} 1$ & Prec & $\operatorname{Rec}$ & $\mathrm{F} 1$ & Prec & $\operatorname{Rec}$ & $\mathrm{F} 1$ \\
\hline word & 31.6 & 52.6 & 39.5 & 59.1 & 59.8 & 59.5 & 37.4 & 31.7 & 34.3 & 52.8 & 53.9 & 53.4 \\
\hline word + path & 32.3 & 53.6 & $40.4^{\bullet}$ & 59.8 & 59.4 & 59.6 & 36.2 & 33.3 & 34.7 & 56.8 & 54.5 & $55.6^{\circ}$ \\
\hline word + pos & 37.1 & 49.3 & $42.3^{\bullet}$ & 57.0 & 60.5 & 58.7 & 38.2 & 39.2 & $38.7^{\bullet}$ & 56.3 & 59.2 & $57.7^{\circ}$ \\
\hline word + path + pos & 37.0 & 44.3 & 40.3 & 66.1 & 52.8 & 58.7 & 37.0 & 37.5 & $37.3^{\bullet}$ & 58.1 & 59.2 & $58.7^{\bullet}$ \\
\hline word + ling & 37.3 & 56.6 & $45.0^{\circ}$ & 53.7 & 70.0 & 60.8 & 49.8 & 41.3 & $45.1^{\circ}$ & 55.6 & 63.1 & $59.1^{\circ}$ \\
\hline word + pos + ling & 38.6 & 53.6 & $44.9^{\circ}$ & 58.0 & 66.7 & $62.0^{\circ}$ & 45.6 & 42.9 & $44.2^{\bullet}$ & 59.8 & 68.2 & $63.7^{\circ \dagger}$ \\
\hline
\end{tabular}

Bold values indicate highest score for SUIT, BENEF, UNSUIT and CAUSE, respectively

Significantly better than word at ${ }^{\circ} p<0.1,{ }^{\bullet} p<0.05$; significantly better than word + ling at ${ }^{\dagger} p<0.1,{ }^{\ddagger} p<0.05$ (based on paired t-test)

related. For the feature side, we could not find any overlapping features. From that we conclude that even though this feature carries some unique information (Table 25), the information that this feature encodes (i.e. that the target food item occurs as a side dish) is just not relevant for the task examined in this article.

\subsection{Sentiment features}

Even though the usage of features from sentiment analysis, or more precisely, polarity classification (Sect. 3.4) seemed very intuitive for this classification task, for none of the different classes to be detected did the usage of those features produce significantly better results than bag of words and the task-specific features (Sect. 3.2), i.e. word and ling in Table 17.

In the following, we try to find reasons for this behavior. We take a closer look at the relation type $B E N E F$ (Sect. 2.3.2). ${ }^{16}$ Intuitively, this type is most likely to correlate with sentiment information, since if some food items are supposed to have some beneficial properties, this should be expressed with some (explicit) positive polar expressions. From the positive relation types we consider (e.g. SUIT, BENEF and PREVENT), BENEF is the relation type with the largest proportion of keywords in the target sentence (Table 16). Having a large proportion of keywords is a prerequisite for a relation type for which sentiment features are effective (sentiment features can be regarded as a subset of keyword features).

We manually annotated all instances labeled as BENEF for which no positive polar expression matched according to our sentiment lexicon. This amounts to $42 \%$ of the utterances. Still, all those utterances express something positive. We distinguish between the following reasons why the sentiment lexicon could not establish this information:

\footnotetext{
${ }^{16}$ We assume that for the other three relation types, i.e. SUIT, UNSUIT and CAUSE, sentiment is not a predictive feature. Causation (as conveyed by CAUSE) is quite different from positive or negative sentiment, so it does not come as a surprise that sentiment features are not effective for this relation type. SUIT and UNSUIT will be discussed in a dedicated section of this error analysis (i.e. Sect. 5.6).
} 


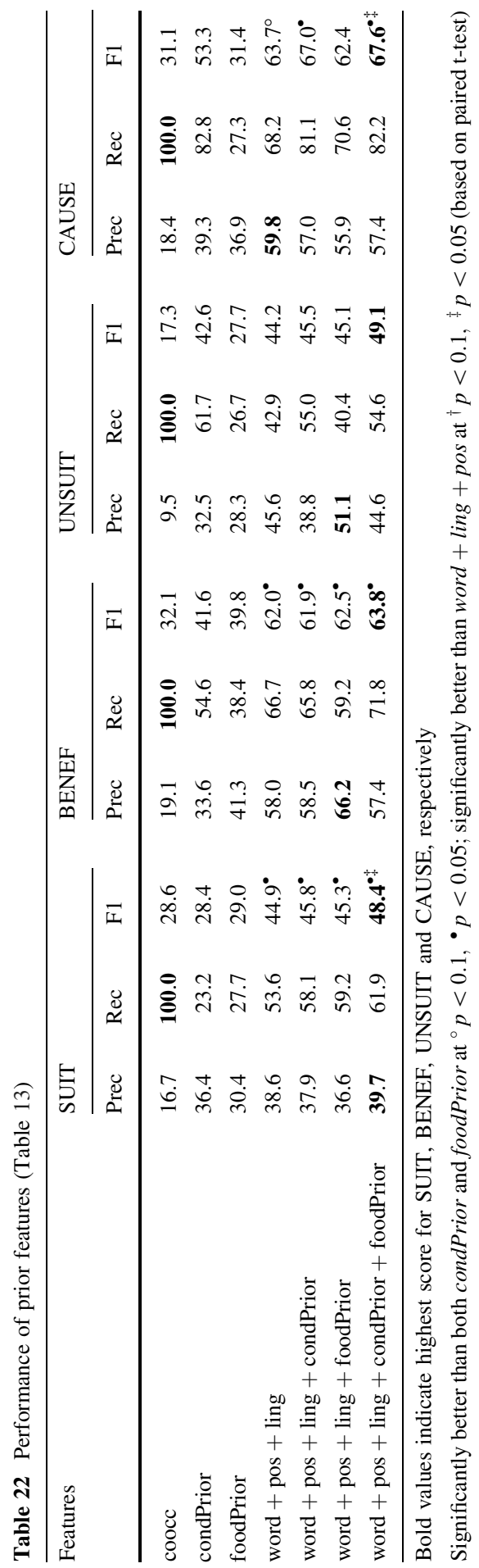


Table 23 Performance of features derived from manually extracted keywords (Table 14)

\begin{tabular}{|c|c|c|c|c|c|c|c|c|c|c|c|c|}
\hline \multirow[t]{2}{*}{ Features } & \multicolumn{3}{|c|}{ SUIT } & \multicolumn{3}{|c|}{ BENEF } & \multicolumn{3}{|c|}{ UNSUIT } & \multicolumn{3}{|c|}{ CAUSE } \\
\hline & Prec & $\operatorname{Rec}$ & F1 & Prec & $\operatorname{Rec}$ & $\mathrm{F} 1$ & Prec & $\operatorname{Rec}$ & F1 & Prec & $\operatorname{Rec}$ & F1 \\
\hline No KW & 39.7 & 61.9 & 48.4 & 57.4 & 71.8 & 63.8 & 44.6 & 54.6 & 49.1 & 57.4 & 82.2 & 67.6 \\
\hline $\mathrm{KW}$ & 41.0 & 64.7 & $50.2^{\dagger}$ & 66.1 & 68.3 & $67.2^{\dagger}$ & 53.5 & 54.6 & 54.0 & 65.6 & 78.5 & $71.5^{\ddagger}$ \\
\hline KW + SyntKW & 43.0 & 61.4 & $50.6^{\dagger}$ & 66.2 & 68.5 & $67.3^{\dagger}$ & 45.8 & 58.3 & 51.3 & 66.4 & 77.0 & $71.3^{\ddagger}$ \\
\hline
\end{tabular}

Bold values indicate highest score for SUIT, BENEF, UNSUIT and CAUSE, respectively

Significantly better than $n o K W$ at $^{\dagger} p<0.1,{ }^{\ddagger} p<0.05$ (based on paired t-test)

(I) There is actually a positive polar expression but it is missing from the sentiment lexicon.

(II) There is actually a positive polar expression but there has been a spelling error or an error in processing (e.g. part-of-speech tagging) so that the positive polar expression could not be matched. ${ }^{17}$

(III) The relation type BENEF is inferred and there is no explicit sentiment in the utterance.

(IV) Some form of negation is involved.

Sentiment analysis is far from being solved. This entails that there does not exist any exhaustive sentiment lexicon. Therefore, it is only natural that errors caused by (I) occur.

There are similar reasons for (II). Tools in natural language processing are known to be error-prone. On user-generated content (as our dataset), errors may not only be caused by the brittleness of NLP software but also by spelling/grammar mistakes in the actual language to be analyzed.

Sentiment does not have to be explicitly expressed. That is, there are utterances that convey some sentiment even though no explicit polar expression is employed (III). Sentence (25), for example, expresses some positive sentiment towards Salbeitee (salvia tea). One can only infer that sentiment with the help of world knowledge. We must know that reducing the level of blood sugar is the goal of a successful treatment of diabetes. Literally speaking, the reduction of the level of blood sugar just denotes a process that can take place within the body of a living being. Therefore, phrases like this are unlikely to be contained in a sentiment lexicon.

(25) Salbeitee senkt bei Diabetes den Zuckerspiegel.

(Salvia tea reduces the level of blood sugar in case of diabetes.)

Finally, we need to address negation (IV). Typically, negation is known to reverse polarity, for instance, a negated negative polar expression can be interpreted as a positive polar expression (as in $\left[\text { not } \mathrm{bad}^{-}\right]^{+}$). This form of negation, however, is not present in our data as far as the relation type $B E N E F$ is concerned. The first difference between the common form of negation and the one that can be found in

\footnotetext{
17 In order to match a polar expression, not only the word token listed in the sentiment lexicon has to match but also its part-of-speech tag.
} 
Table 24 Frequency of effective/less effective linguistic features

\begin{tabular}{llc}
\hline Feature & Frequency & Percentage of Corpus \\
\hline less effective features & & \\
scope & 819 & 32.3 \\
weird & 280 & 11.1 \\
side & 222 & 8.8 \\
question & 180 & 7.1 \\
effective features & & \\
negFood & 384 & 15.2 \\
synoHlthTS & 292 & 11.5 \\
causeTS & 183 & 7.2 \\
againstCond & 180 & 7.1 \\
\hline
\end{tabular}

sentences labeled with our target relation types is that we do not encounter ordinary polar expressions involved in these constructions, but health conditions. Health conditions, if they represent some illness (e.g. headache or cancer), can also be considered as a negative polar expression. These expressions are not systematically listed in our sentiment lexicon (approximately $25 \%$ of them are missing in our German lexicon). Given that our list of health conditions is known in advance, however, this problem could be easily solved by adding all of those health conditions to our sentiment lexicon. What makes this form of negation really difficult is that in almost $90 \%$ of the cases, negation is not conveyed by typical negation words [e.g. kein (no), nicht (not)]. Instead, so-called shifters (Wilson et al. 2005 ), i.e. (predominantly) verbs, nouns and adjectives whose meaning is similar to negation expressions (e.g. bekämpfen (combat), lindern (abate)), are used. ${ }^{18}$ Unfortunately, the means to form negations with shifters are pretty diverse, since there is a large set of shifters (26)-(29). ${ }^{19}$ Due to the fact that there do not exist any robust lexicons of shifters ${ }^{20}$, we could not properly model this phenomenon with one exception. While most individual shifters occurred only very rarely, there was one shifter against which occurred very often (29), which lead us to the task-specific feature againstCond (Table 6).

(26) Nach zwei Tassen Tee sind meine Halsschmerzen schon fast $\mathbf{w e g}_{\text {shifter }}$. (After two cups of tea, my sore throat is almost gone $_{\text {shifter }}$.)

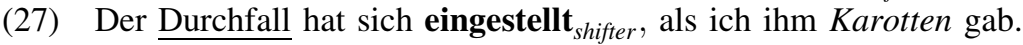
(His $\underline{\text { diarrhoea }}$ subsided shifter $_{\text {after I gave him carrots.) }}$

\footnotetext{
${ }_{18}$ Please note that the situation is different in the case of UNSUIT, where common negation words predominate (Sect. 5.6, Table 29).

19 More than $60 \%$ of the shifters occur as singletons on the sentences labeled as BENEF.

20 The shifter lexicon from Wilson et al. (2005) just contains 67 shifters which, when translated to German, would have only a marginal impact on our dataset.
} 
Table 25 Feature overlap of less effective features with other features (we only list features with a correlation of Chi-square 50 or higher)

\begin{tabular}{llc}
\hline Feature & Correlated Features & Chi-Square Score \\
\hline question & boundary & 579.7 \\
scope & negFoodSynt & 98.9 \\
& boundary & 85.4 \\
& sNegFoodSynt & 60.9 \\
side & no strongly correlated features & N/A \\
weird & foodBefCond & 113.0 \\
\hline
\end{tabular}

(28) Die furchtbaren Kopfschmerzen werde ich jetzt mal mit Kaffee bekämpfen $_{\text {shifter }}$.

(I will now combat shifter $_{\text {my dreadful headache with coffee.) }}$

(29) Gegen shifter $_{\text {den Durchfall kannst Du Bananen essen. }}$

(You may eat bananas against shifter $_{\text {your diarrhoea.) }}$

Table 26 displays the distribution of the different cases in which no positive polar expression according to the sentiment lexicon matched. By far the most frequent case is the shifter based on against, i.e. againstCond. This is also consistent with our evaluation of task-specific features in Table 18 where againstCond is the second strongest feature. Other frequent cases in Table 26 are negations other than againstCond and unigram polar expressions missing from our lexicon. These polar expressions form a subset of manually chosen keywords (Sect. 3.7) and, indeed in Table 23, we saw a significant improvement on $B E N E F$ caused by adding all manually chosen keywords. The remaining cases are fairly difficult to cope with, even if we had better lexical resources. This also concerns the case when only a polar expression in the context is present (but not the target sentence). If a polar expression appears in one of the two sentences preceding or following the target sentence, it is pretty difficult to decide whether this mention refers to the relation between food item and health condition expressed in the target sentence. So far, there do not exist reliable means in NLP to establish intersentential relationships. Multiword expressions could also be covered by a better sentiment lexicon, however, with a proportion of only $2.9 \%$, it would be unlikely to significantly increase classification performance.

While Table 26 displays all cases in which no (positive) polar expression from our sentiment lexicon matched, for $58 \%$ of the sentences labeled as BENEF in our gold standard, there is a match. Yet, using polar expressions does not significantly improve classifiers trained on bag of words (Table 17). We will examine the role of bag-of-words features in the next section and try to find a reason why it is so difficult to beat that baseline on our dataset.

\subsection{Bag of words}

Our previous evaluation (Tables 17,21) established that bag of words poses a strong baseline. On the other hand, several features that are based on a list of cue words 
(e.g. polarCont from Table 8) did not have the expected impact on classification (at least not when added to bag of words). The reason for this may be that bag of words captures a considerable amount of the information that is contained in such word-list features. This is particularly true if there are some frequent (discriminatory) cue words. By visual inspection we found that for some relation types there are some recurring language patterns, e.g. for BENEF, $X$ hilft gegen $Y$ (X helps against $Y$ ) or for CAUSE, von X bekomme ich $Y$ (I get $Y$ from $X)$. Sentences (30)-(36) illustrate this for the relation type $B E N E F$. Inevitably, with bag of words, a classifier will pick up correlations, for instance, a correlation between (the positive polar expression) hilft and BENEF or between bekomme and CAUSE. Moreover, we also assume that the type of domain we analyze may additionally support the effectiveness of such features. Users that post entries in food-related forums rarely pay much attention to stylistic diversity. In other words, it does not matter greatly to them if certain formulations are constantly repeated. This situation is certainly different in other text types, such as news editorials or movie reviews ${ }^{21}$ where the authors are also more inclined to appeal to the readers with their language and, therefore, make use of a more diversified vocabulary.

(30) Natron hilft gegen Sodbrennen.

(Baking soda helps against heartburn.)

(31) Bei Übelkeit und Erbrechen hilft Ingwer.

(Ginger helps in case of nausea and vomiting.)

(32) Honig hilft sehr gut bei Husten.

(Honey helps very much in case of cough.)

(33) Holundersaft ist gesund und hilft gegen Fieber.

(Elderberry juice is healthy and helps against fever.)

(34) Kaffee hilft mir auch oft bei Kopfschmerzen.

(Coffee also often helps me when I got a headache.)

(35) Mich hat die Grippe erwischt, da hilft nur Hühnersuppe.

(The flu got me, only a chicken broth will help.)

(36) ... und auch bei Bauchweh und Durchfall hilft Cola.

(... and even in case of stomach ache and diarrhoea, coke will help you.)

In order to substantiate our intuition regarding the relationship of our taskspecific features and bag of words, we extracted for the strongest task-specific features (throughout the different classes) the words (unigrams) most strongly correlated with them according to Chi-square statistics. The results are displayed in Table 27. Indeed, there are some very frequently occurring unigrams, such as helfen (help), gut (good) or verursachen (cause). Our cue-word lists, such as our sentiment lexicon or cause-cues, become less important in the light of such expressions. Apparently, there is really little lexical diversity on our dataset, which means that bag-of-words classifiers can easily pick up the importance of particular words towards the classes to be predicted.

\footnotetext{
${ }^{21}$ We mention these text types since some of our word-list features, such as polar expressions, have successfully been applied to such domains and are known to improve bag-of-words baselines (Wilson et al. 2005; $\mathrm{Ng}$ et al. 2006).
} 
The table also shows that different features (e.g. synoHlthTS or againstCond) overlap with the same words, i.e. helfen (help) or gegen (against). This is an indication that even though the features are meant to model different things, to some extent they capture the same information. ${ }^{22}$ However, one should not conclude from Table 27 that features, such as synoHlthTS, negFood or posCondSynt, are identical. In fact, we have proven the opposite in Table 20 by computing the most predictive subset of complementary features using a best-forward subset selection. (For several classes, these different features are contained in the resulting best-forward subset, which means that they are, at least to some extent, complementary.)

Several of our features also express a conjunction of different properties. For instance, negFood indicates a mention of a food item that is also negated. Similarly, posCondSynt conveys the occurrence of a positive polar expression that is also syntactically related to some health condition. However, these kinds of features still preserve a very high correlation towards particular words. From that we conclude that to a large extent, the information contained in those features mainly stems from the expressiveness of individual (high-frequency) words rather than the cooccurrence with some additional linguistic property. For example, as far as negFood is concerned, the mere presence of simple negation expressions within a sentence may already reveal a substantial amount of information as to which type of relation between the health condition and the target food item holds.

In Sect. 4.1.2, we already pointed out the strength of the feature foodBefCond compared to other task-specific features. The analysis displayed in Table 27 additionally confirms that the information encoded cannot be expressed by a subset of words, as no word strongly correlates with this feature. Therefore, foodBefCond is a feature that is pretty much unique in the information that it encodes.

\subsection{Bag of words versus syntactic features}

In our evaluation, we employed two variations for some features: one plain feature and the other combined with some syntactic restriction. For instance, for the features derived from the sentiment lexicon (Table 8), we included the plain features (polarCont) and the features where food item and polar expression also have to be syntactically related (polarSynt). Similarly, we divided the set of features using the keyword lexicon (Table 14) into the plain keyword features $(K W)$ and the features for which the keyword has to be either syntactically related to the target food item or the health condition (SyntKW).

The effectiveness of these syntactically restrained features is somewhat mixed (Tables 18, 23). In the case of the sentiment features (Table 18), there is a general limitation that sentiment expressions only strongly correlate with one relation type, i.e. $B E N E F$. However, for that particular relation type, the strongest syntactic features are much more effective than the strongest plain features (i.e. sPosCondSynt/posCondSynt with Chi-square scores of more than 200 vs. sPosTS/

\footnotetext{
22 The counterintuitive result that helfen (help) appears on list of againstCond and gegen (against) appears on the list of synoHlthTS can be explained by the fact that the two words basically form a collocation helfen gegen (help against) (see also Sentences (30) and (33)).
} 
Table 26 Statistics of utterances labeled as $B E N E F$ where no positive polar expression matched according to the sentiment lexicon

\begin{tabular}{ll}
\hline Category & Percentage \\
\hline againstCond & 32.2 \\
negation/shifter (except against) negating health condition (proportion of shifters: & 17.6 \\
$90 \%$ ) & \\
unigram positive polar expression missing from sentiment lexicon & 17.6 \\
no positive polar expression at all & 16.6 \\
no positive polar expression in target sentence but there is some positive polar & 11.2 \\
expression in the remaining context & \\
multiword positive polar expression (missing from sentiment lexicon) & 2.9 \\
spelling error/error in processing & 2.0 \\
\hline
\end{tabular}

sNegTS with Chi-square scores of just above 90). For the keyword features, we could not find any evidence that the syntactic features significantly improve the plain features (Table 23).

As far as the syntactic keyword features $(\operatorname{Synt} K W)$ are concerned, we consider it unlikely that the lacking effectiveness is due to flaws in the feature extraction. This is since the underlying resource (the Stanford parser for German) for establishing whether two words are syntactically related is also employed for the syntactic sentiment features, which have been found effective (e.g. sPosCondSynt or posCondSynt in Table 18).

Complementary to the analysis of the keyword and sentiment features, we also examined generic linguistic features (Table 7) which exclusively encode syntactic knowledge. However we distinguish between features incorporating part-of-speech information and features encoding information from a syntactic parse tree. The evaluation of those features (Table 21) revealed that part-of-speech information is more effective than information derived from the syntactic parse tree. This result mirrors the only mild effectiveness of the syntactic keyword/sentiment features. Those features, too, incorporate information from a syntactic parse tree rather than part-of-speech information.

In general, syntactic features do not always have to outperform shallow/bag-ofwords features. A detailed study for text classification (on the document level) examining various datasets was presented by Moschitti and Basili (2006). Since we obtain similar results on German data as Moschitti and Basili (2006) do on English data (i.e. their syntactic features do not help on that task), we conclude that our results indicating minor effectiveness of many syntactic features cannot be (exclusively) ascribed to the fact that we examine German language data (German NLP-tools are known to be less robust than their English counterparts).

A further noteworthy recent example for lacking effectiveness of syntactic features is the NIST benchmark on slot-filling for knowledge-based population from 2013, in which the top-scoring system (Roth et al. 2014) did not incorporate syntactic features while many of the other participating systems did. Roth et al. (2014) also carry out ablation studies with syntactic features on their system further 
supporting that, on the task of slot-filling for knowledge-based population, such features are less helpful.

\subsection{The difficult relation types: SUIT and UNSUIT}

Throughout our experiments, we found that the two classes SUIT and UNSUIT score consistently lower than the other two classes BENEF and CAUSE. We found the following reason for that:

For both SUIT and UNSUIT, there are a lot fewer predictive lexical cues than for $B E N E F$ and CAUSE. Three individual results of our experiments support that claim. Firstly, for SUIT and UNSUIT, the proportion of keywords manually annotated (Table 16) is much smaller, i.e. 21.73 and 32.39, than for the other two classes, i.e. 56.97 and 72.20. Secondly, among the set of effective linguistic features (Table 18), only for BENEF and CAUSE were there lists of cue words, i.e. synoHlthTS, synoHlthEC, sPosCondSynt and posCondSynt for BENEF, and causeTS and causeEC for CAUSE. Thirdly, the bag-of-words feature set $^{23}$ (Table 17) produces much higher F-scores for BENEF (59.4) and CAUSE (53.4) than for SUIT (39.5) and UNSUIT (34.3).

For most of those cases labeled as either SUIT or UNSUIT where no keyword is present, we did not find any obvious and recurring linguistic pattern that one could use. For most of these sentences, one has to infer the pertaining relation. In Sentence (37), for example, it is obvious to the reader that the speaker's grandmother drinks that much mineral water since her doctor told her to do so because of her diabetes. However, this is extremely difficult to recognize automatically. Likewise, in Sentence (38), one must have the knowledge that acidic food should be avoided if one suffers from heartburn. ${ }^{24}$ The speaker of the utterance recommends adding sugar to tomato sauce as a means to neutralize the acidity of tomatoes. From that one must infer that if this special trick was not applied, tomatoes would contain too much acid. From that we conclude that, plain tomatoes are unsuitable for people suffering from heartburn.

(37) Meine Oma hat nun Diabetes und ihr hängt das Mineralwasser schon zum Hals raus.

(My grandma suffers from diabetes and is completely fed up with drinking mineral water.) LABEL: SUIT

(38) Noch ein Tipp für Leute mit Sodbrennen: etwas Zucker in die (Tomaten-) Sauce geben, das nimmt die Säure der Tomaten.

(Here is a tip for people with heartburn: add some sugar to the (tomato) sauce, it will reduce the acid of the tomatoes.) LABEL: UNSUIT

To further substantiate that the classes SUIT and UNSUIT are difficult and that the features we previously examined are, in principle, correctly implemented, but just do not sufficiently correlate with those classes, we manually annotated the

\footnotetext{
23 We consider our unigram bag-of-words features as a typical example of lexical information.

24 Note that we do not consider acid as a negative polar expression. Acid is not harmful per se. (For example, our digestive system depends on gastric acid.) This is also reflected by the fact that it is not contained in our sentiment lexicon.
} 
Table 27 Words that highly correlate with the strongest task-specific features

\begin{tabular}{|c|c|}
\hline Feature & Words \\
\hline synoHlthTS ${ }^{\mathrm{a}}$ & helfen (help) $[1380.5,169]$; gegen (against) $[157.7,85]$; gesund (healthy) $[84.0,12]$ \\
\hline againstCond $^{\mathrm{a}}$ & gegen (against) $[1928.4,180]$; helfen (help) $[149.8,52]$ \\
\hline negFood $^{\mathrm{a}}$ & $\begin{array}{l}\text { nicht (not) }[273.6,111] \text {; kein (no) }[273.3,102] \text {; ohne (without) }[158.9,42] \text {; verzichten } \\
\quad \text { (do without) }[73.1,20]\end{array}$ \\
\hline sPosCondSynt ${ }^{\mathrm{b}}$ & helfen (help) [664.7, 128]; gut (good) [190.1, 74]; gegen (against) [184.6, 95] \\
\hline posCondSynt ${ }^{b}$ & helfen (help) [453.3, 131]; gegen (against) [143.8, 106]; gut (good) [136.3, 79] \\
\hline causeTS ${ }^{\mathrm{a}}$ & $\begin{array}{l}\text { verursachen (cause) }[667.7,52] \text {; führen (lead to) }[336.6,27] \text {; auslösen (trigger) [141.2, } \\
\text { 12]; zu (to) [77.6, 49]; können (can) }[67.1,35] \text {; auftreten (occur) }[64.0,6]\end{array}$ \\
\hline foodBefCond $^{\mathrm{a}}$ & no strongly correlated words present \\
\hline
\end{tabular}

The numbers in square brackets in the column Words denote the Chi-square score of each word towards the respective feature and the frequency of co-occurrence with that feature; we only list features with a Chi-square score of 100 or higher from Table 18; we only list words with a correlation towards the taskspecific feature of Chi-square 50 or higher; in case of task-specific features that only marginally differ in scope, e.g. synoHlthTS and synoHlthEC, we only list the stronger feature in this table

${ }^{\text {a }}$ Linguistic features (Table 6)

b Sentiment features (Table 8)

sentences labeled as either SUIT or UNSUIT. We assign a label indicating the most predominant cue that indicates to a human annotator that the particular class label is present. In this annotation, we annotate linguistic categories rather than just the presence of lexical cues (Table 16). ${ }^{25}$ We consider polar expressions (both contained and not contained in our sentiment lexicons), negation (we distinguish between the negation words and further shifters (cp. Sentences (26)-(29) in Sect. 5.3)), discourse relations indicating causal/conditional relations (anchored by an explicit discourse connective, e.g. wenn (if) or weil (because)) ${ }^{26}$, and lexicosyntactic cues (involving neither negation nor other types of lexical items mentioned before in this list). Finally, there are also sentences in which inferences are necessary (e.g. Sentences (37) or (38)).

The results for SUIT are displayed in Table 28 and for UNSUIT in Table 29, respectively. ${ }^{27}$

The distribution of the categories is consistent with our previous evaluation. As far as SUIT is concerned, half of the sentences require human inferencing. The only other frequent cues are discourse relations with explicit discourse connectives. We subsequently tried to automatically detect these relations, however, we failed to

\footnotetext{
25 Unlike the lexical cues, some of our categories also consider additional syntactic information.

26 Example: I eat almonds because I suffer from dermatitis.

27 Notice that there is no direct correspondence between the categories from Table 16 stating the proportion of instances with manually annotated keywords and Tables 28 and 29. Table 16 only considers unigram keywords being either nouns, verbs or adjectives while the annotation in Tables 28 and 29 is unrestricted. In other words, keywords annotated in Table 16 are not the sum of categories excluding inferences in Tables 28 and 29. There will also be other constructions marked in those tables that were not captured by the restricted annotation from Table 16.
} 
Table 28 Manual annotation of predictive cues among the sentences labeled as SUIT

\begin{tabular}{lc}
\hline Cue & Percentage \\
\hline inferences & 49.5 \\
discourse relations (with explicit discourse cue, e.g. weil (because)) & 21.0 \\
lexico-syntactic cues & 15.7 \\
polar expression (contained in sentiment lexicon) & 6.1 \\
polar expression (not contained in sentiment lexicon) & 4.4 \\
uncovering relation requires consideration of further context & 2.3 \\
$\quad$ (i.e. preceding/following sentence(s)) & \\
negation word (with complex construction involving health condition/food item) & 1.0 \\
negated polar expression & 0.0 \\
food item negated by negation word & 0.0 \\
food item negated by shifter & 0.0 \\
shifter (with complex construction involving health condition/food item) & 0.0 \\
\hline
\end{tabular}

Table 29 Manual annotation of predictive cues among the sentences labeled as UNSUIT

\begin{tabular}{lc}
\hline Cue & Percentage \\
\hline food item negated by negation word & 42.1 \\
inferences & 18.2 \\
food item negated by shifter & 11.3 \\
negation word (with complex construction involving health condition/food item) & 8.9 \\
negated polar expression & 6.5 \\
polar expression (contained in sentiment lexicon) & 4.9 \\
shifter (with complex construction involving health condition/food item) & 2.4 \\
discourse relations (with explicit discourse cue, e.g. weil (because)) & 2.0 \\
polar expression (not contained in sentiment lexicon) & 1.6 \\
uncovering relation requires consideration of further context & 1.2 \\
(i.e. preceding/following sentence(s)) & \\
lexico-syntactic cues & 0.0 \\
\hline
\end{tabular}

improve performance. One reason for this is that German discourse connectives are ambiguous (e.g. da (because/there)).

As far as UNSUIT is concerned, we see further evidence that negation is, by far, the most important linguistic phenomenon to be addressed. Furthermore, it is the case that the target food item being negated is the most relevant form of negation. The table also shows that we miss some forms of negation, in particular, those involving shifters, i.e. many words not contained in our negation lexicon. There is still a significant amount of cases which require inference, but it is considerably lower than for SUIT. Unlike SUIT, other forms of lexical cues not involving polar expressions or negation do not seem to play a role for UNSUIT. 
A comparison of Tables 28 and 29 may suggest that it should be easier to detect the relation type UNSUIT than the relation type SUIT. Still, in our evaluation we obtained similar F-scores for both classes (Tables 17, 21, 22, 23). The reason for this may lie in the class distribution (Table 3). From a feature perspective, UNSUIT may be easier to handle, however, this effect is less visible in our evaluation since UNSUIT is much rarer $(9.49 \%$, Table 3$)$ than SUIT (16.44 \%, Table 3). The latter occurs almost twice as often as the former. Typically, it is more difficult to detect rare classes (i.e. UNSUIT) than frequently observed classes (i.e. SUIT).

Despite the fact that SUIT and UNSUIT score much lower in comparison to $B E N E F$ and CAUSE, we assume that further significant improvements could be achieved in the presence of more training data. Figure 1 displays the learning curve using the full feature set (including even keywords), i.e. KW from Table 23. The figure suggests that at least the curves for SUIT and UNSUIT are far from being saturated (in contrast to $B E N E F$ ). ${ }^{28}$ (We assume that the more training data is added, the more information can be learned about less frequent lexical features.) Still, one may doubt that given sufficient training data, one will ever obtain scores similar to those obtained for BENEF or CAUSE.

In this error analysis we established that a very large amount of instances labeled as SUIT require some form of inference (Table 28). These sentences are unlikely to be resolved with current NLP technologies. At this point, we should wonder whether it should be equally important to extract all types of sentences automatically that express our target relations. In Sect. 1, we briefly sketched as a potential application of our task an intelligent search engine for forum entries. Such a component would typically be used by laymen possessing no knowledge about the algorithms that underlie the search engine. As a consequence, the sentences extracted should be easy to verify. Examples where the target relation is present but must be inferred, such as Sentences (37), may not be perceived as particularly convincing to such users. Explicit alternatives, such as Sentence (39) should be preferable. Such alternatives contain some form of explicit (lexical) cues and are much more likely to be extracted automatically. Therefore, we think it does not matter too greatly that some relation instances (i.e. the implicit ones requiring inference) cannot be extracted automatically.

(39) Bei Diabetes empfehle ich Mineralwasser.

(For diabetes, I recommend mineral water.) LABEL: SUIT

\subsection{Textual quality of the corpus}

Since our text corpus has been extracted from user-generated content of the web, we need to address its textual quality. Obviously, we cannot expect the level of correctness encountered on newspaper corpora that are typically employed for NLP

\footnotetext{
28 It may come as a surprise that the classes $B E N E F$ and CAUSE already produce high scores with only $25 \%$ of the training data. This is due to the fact that we consider the full feature set that includes many features based on word lists. Typically, such features are particularly effective if only few training data are present. Such features generalize over individual word occurrences and are less sparse than bag of words.
} 


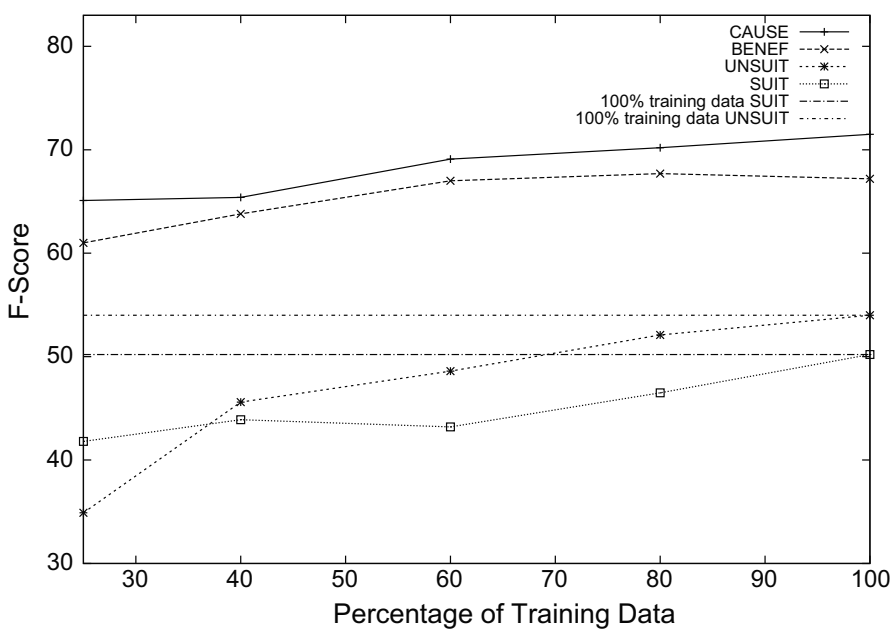

Fig. 1 Learning curve for the different classes using the full feature set including keywords (i.e. KW from Table 23)

tasks. In order to measure the amount of noise in our dataset, we randomly sampled 100 sentences from our corpus in which a food item and some health condition cooccurred and manually annotated the types of errors that were encountered.

Table 30 lists the results of this evaluation. With regard to grammar, we distinguished between obvious errors and non-standard grammatical constructions. For instance, we encountered many sentences in which the subject is missing (40). Even though such constructions are considered ungrammatical in formal written language, they are acceptable and commonly found in informal language (including spoken language).

(40) Heute bleibe ich bei Zwieback; hatte letzte Nacht furchtbares Sodbrennen. (Today, I stick to zwieback; had a terrible heartburn last night.)

We also added very informal expressions to our list (including informal abbreviations, such as $v$ ll for vielleicht (perhaps)), despite the fact that they are not errors, strictly speaking. Still, they are a potential error source for part-of-speech tagging or syntactic parsing since they are treated as unknown words. ${ }^{29}$

Table 30 confirms that there is a significant amount of errors present in our dataset. These errors are also likely to affect our automatic extraction procedure.

One notable error source that has been caused by pre-processing our text corpus are errors in sentence boundary detection which occur in $25 \%$ of the sentences. We used a standard tool for German, i.e. the German model from $O p e n N L P^{30}$, and are not aware of a more recent/robust tool, so there are no realistic alternatives for this processing step that could reduce those errors significantly.

\footnotetext{
${ }^{29}$ The training data for those tools typically originate from the news domain, where such expressions cannot be found.

${ }^{30}$ opennlp.sourceforge.net/projects.html.
} 
Table 30 Distribution of error types manually annotated on a random sample of 100 sentences (a sentence may contain several errors at the same time)

\begin{tabular}{lc}
\hline Error Type & Percentage \\
\hline non-standard grammatical construction & 26 \\
incorrect sentence boundary detection & 24 \\
spelling error & 19 \\
punctuation error & 16 \\
very informal/non-standard wording & 16 \\
tokenization error & 12 \\
grammatical error & 6 \\
\hline
\end{tabular}

In general, most of the errors are inherent to this text type. In order to better handle those domain idiosyncrasies, it would be worthwhile to employ a part-ofspeech tagger and/or a syntactic parser which has been specially trained on such social media texts containing informal language. However, such an undertaking is beyond the scope of the research presented in this article. Neither is there a publicly available tool of that sort for German.

Despite the present formal errors contained in our text corpus, we still think that our resource is appropriate for this research. The amount of spelling mistakes is actually low. We could hardly find any health conditions/diseases or drugs (which can be quite complex) written incorrectly. Most of the errors are simple typos. The amount of grammatical errors (excluding non-standard usage) is even lower than the amount of spelling mistakes. Given these observations, our text corpus is unlikely to represent a particularly poor choice of language data. Further, it is very unlikely that there exists a similar alternative textual source from the web with a similar coverage on the issues we want to extract.

\subsection{Fine-grained versus coarse-grained analysis}

In this section, we contrast the fine-grained classification that we examined in the previous sections with a more coarse-grained classification. Rather than addressing four different classes, we could alternatively merge the four classes into two large classes, i.e. one positive class (combining SUIT and BENEF) and one negative class (combining UNSUIT and CAUSE). Through this experiment, we want to provide further evidence that our fine-grained class inventory is well-defined. This would not be the case if it were significantly easier to just distinguish between two strongly opposing classes and thus achieve a much higher classification performance (than on the fine-grained set-up). This would indicate that the fine-grained classifiers confuse the different classes too much.

Table 31 compares the performance of the fine-grained and coarse-grained setting. We compare two different classification approaches: individual considers fine-grained classes, that is, in the training data there are four classes (to be predicted); in merged, there are only two coarse-grained classes (i.e. SUIT + BENEF and UNSUIT + CAUSE). In order to have a meaningful 
Table 31 Performance on the coarse-grained classes (i.e. SUIT + BENEF and UNSUIT + CAUSE)

\begin{tabular}{llllllll}
\hline Classifier & \multicolumn{3}{l}{ SUIT + BENEF } & & \multicolumn{3}{l}{ UNSUIT + CAUSE } \\
\cline { 2 - 3 } & Prec & Rec & F1 & & Prec & Rec & F1 \\
\hline individual (fine-grained) & 61.72 & 75.36 & 67.86 & & 64.26 & 71.87 & 67.82 \\
merged (coarse-grained) & 60.60 & 82.10 & $69.73^{\circ}$ & & 57.16 & 78.61 & 66.19 \\
\hline
\end{tabular}

- Significantly better than individual at $p<0.05$

comparison, we must produce a common output format for both classification approaches. Therefore, we converted the output of the fine-grained classifier to the output of the coarse-grained classifier. So, even though individual originally predicts four different classes, after conversion, there are only two class labels that are predicted (either prediction of SUIT and BENEF is converted to the coarsegrained class SUIT $+B E N E F$, while either prediction of UNSUIT or CAUSE is converted to the coarse-grained class UNSUIT + CAUSE).

If our fine-grained class inventory were inappropriate, that is, we were artificially separating the two coarse-grained classes into two halves each, then we would end up having very similar (perhaps even almost identical) instances in two separate (fine-grained) classes. Such partition of instances would become fairly problematic for a fine-grained classifier because it would be forced to learn inconsistent information (i.e. two similar instances belonging to different classes). The result of this would be that the fine-grained classifier (individual) performs much worse than the coarse-grained classifier (merged). (The latter should be better since it would be trained on meaningful classes.) If, however, the fine-grained class inventory is appropriate (this means that the different classes would contain sufficiently different instances), the result would be that there is no notable difference between merged and individual.

Table 31 shows that for the two positive classes, i.e. SUIT and BENEF, the classifier trained on a merged positive class performs slightly better than the classifier trained on the individual classes. The difference between individual and merged is even statistically significant. However, for the negative classes there is no such effect. From that we conclude that SUIT and BENEF are more similar to each other than UNSUIT and CAUSE. A fine-grained classifier has a tendency to confuse the two positive classes. However, given that there is only a slight decrease in performance by individual on SUIT $+B E N E F$, we do not think that our fine-grained class inventory is insufficiently well-defined.

\section{Related work}

In the health/medical domain, the majority of research focuses on domain-specific relations involving entities, such as genes, proteins and drugs (Cohen and Hersh 2005). More recently, the prediction of epidemics from social media (Fisichella et al. 2011; Torii et al. 2011; Diaz-Aviles et al. 2012; Munro et al. 2012) has attracted the attention of the research community. In addition, there has also been 
work on processing health-care claims (Popowich 2005) and detecting sentiment in health-related texts (Sokolova and Bobicev 2011).

Research in the food domain using natural language processing has so far focused on the following tasks: The most prominent research addresses ontology or thesaurus alignment (Hage et al. 2010), a task in which concepts from different sources are related to each other. In this context, hyponymy relations (van Hage et al. 2005) and part-whole relations (van Hage et al. 2006) have been explored. More recently, in Chahuneau et al. (2012) sentiment information has been related to food prices with the help of a large corpus consisting of restaurant menus and reviews. In Wiegand et al. (2012b), extraction methods for domain-specific relations in the food domain have been examined. The relations that are dealt with are motivated by customer needs in a supermarket. These relations answer the questions which food items can be consumed together, which food items are typically offered on a particular social event and which food items can be substituted by each other. Unlike this work, where individual utterances are evaluated, Wiegand et al. (2012b) exclusively evaluate relation extraction based on an aggregate of multiple utterances. The extraction methods employed, i.e. lexical surface patterns and statistical co-occurrence, are fairly simple and require little linguistic processing. Wiegand et al. (2012a) is an extension of Wiegand et al. (2012b) in which those extraction methods are tested on different corpora, thus showing that domain-specific texts are necessary in order to obtain reasonable performance. Moreover, the different extraction methods are combined. Wiegand et al. (2014) examine the extraction of individual utterances in a supervised learning scenario and incorporate common food categories (e.g. fruits, meat, vegetables etc.) as features.

Our work also bears some relation to the recent work on sentiment analysis addressing goodFor/badFor events, i.e. events that have a positive or negative effect on the entities involved in them (Deng and Wiebe 2014; Deng et al. 2014). Food items could also be interpreted as such events, while the persons with specific health conditions are the entities on whom the food items have either a positive or negative impact. Rather than looking at explicit cues, as we do, Deng and Wiebe (2014) and Deng et al. (2014) largely focus on opinion implicatures, i.e. (defeasible) implicit opinions, that are uncovered by automatic inference. There are several reasons, however, why we think that this approach cannot be immediately applied on our task setting. Firstly, the text source examined for goodFor/badFor events are editorials or blogs. These texts are longer monothematic discourses where there is much context with explicit sentiment information to infer the relation involving goodFor/badFor events. Our contexts are much shorter and largely multi-topic (as a matter of fact, several relations expressing the suitability or unsuitability of food items are expressed as a side note) where such inference mechanism is likely to fail. Secondly, their work relies on a gold annotation of explicit sentiment (which is used to infer the implicatures). In realistic situations that kind of information is not available, and automatic methods to detect it are still fairly error-prone. Moreover, on our task, we found that there is only a strong correlation between explicit sentiment (i.e. polar expressions) and one out of four classes we want to extract (i.e. $B E N E F)$. 
The work that is most closely related to this article is Wiegand and Klakow (2013a) in which the contextual healthiness of food items is extracted from natural language texts. In both this article and Wiegand and Klakow (2013a), some contextual analysis is carried out on sentences that contain (hopefully healthrelated) mentions of food items. The fundamental difference to this article is that in Wiegand and Klakow (2013a) the general or prior healthiness of food items is to be determined instead of conditional healthiness. In Sect. 3.5, we explained the difference between these two concepts in detail. It has also a notable impact on the usability of resources. As far as general/prior healthiness is concerned, a healthiness lexicon can be used as a source for prior features. Indeed, Wiegand and Klakow (2013a) confirm a strong correlation between the content of the healthiness lexicon and the information contained in their text corpus. In this article, however, such a resource was considered less useful. Wiegand and Klakow (2013a) and this article also work with different instance spaces: While Wiegand and Klakow (2013a) consider sentences with co-occurrences of the word healthy and some food item, in this article, we consider sentences in which food items co-occur with mentions of particular health conditions. (Due to the different instance spaces, different feature sets are also applied in these two different scenarios.) Prior healthiness is a binary classification problem (i.e. either a food item is healthy or not) while in this article we identified several subcategories of suitability and unsuitability which we also tried to separate. Wiegand and Klakow (2013a) spend some considerable effort in finding reliable utterances regarding healthiness. As far as conditional healthiness is concerned, we do not filter these cases (e.g. hedging), as most relations between food items and health conditions are weak and thus associated with a relatively high degree of uncertainty. Wiegand and Klakow (2013a) also experiment with different types of classifiers, including aggregate-based and rule-based classification, while in this work we focus on feature engineering for supervised learning.

Finally, Wiegand and Klakow (2013b) examine the problem of detecting reliable food-health relationships. Their work also carries out experiments on the dataset examined in this article. The focus of that work is that given a food-health relationship, how can one decide automatically whether that information is perceived as reliable or not. Wiegand and Klakow (2013b) already assume the relationships between food items and health conditions as given (they just read off the labels from the gold-standard), while this article focuses on how these labels can actually be automatically detected.

\section{Conclusion}

We presented a new annotation scheme for conditional healthiness extraction, that is, the extraction of suitable and unsuitable food items regarding specific health conditions. Rather than considering this task as a binary classification problem we also considered subtypes of suitability and unsuitability.

We examined a plethora of features for supervised classification and found that task-specific resources, such as a healthiness lexicon classifying food items according to prior healthiness, do not systematically help for this task. Instead a 
feature set comprising bag-of-words features, some task-specific linguistic features and part-of-speech information produces a much better classifier. The task-specific linguistic features we include focus on three groups of features, those being configurational cues implying that the target food item and health condition are related to each other, features checking whether the assumed relation is embedded in a context that invalidates the relation (e.g. negation, irrealis etc.) and some lookup lists containing words indicative of concepts that are relevant for this task.

We also found that some individual food items and health conditions correlate with particular relation types. Using this knowledge as stand-alone prior features mostly outperforms a trivial co-occurrence-based baseline and can also be usefully combined with the remaining features for supervised learning.

A further result of our research is that, to some extent, the four different classes we examine display different properties and therefore show different effectiveness with regard to specific features:

SUIT is the most difficult class producing the lowest overall scores. Apart from the features that basically help for all classes, e.g. generic linguistic features, prior features or only manually chosen keywords, we could not determine any particularly outstanding feature. Most surprisingly, polar expressions do not correlate with this class. The learning curve from that class, however, indicates that, given more labeled training data than are provided by the current dataset, classifiers with better performance could be achieved. This class contains a high number of instances where some inference is required. Prospective applications incorporating the extraction of conditional healthiness may not suffer too greatly from the fact that those implicit relation instances cannot be reached, since users may find explicit instances more convincing.

$B E N E F$ behaves very differently from SUIT, even though they both convey suitability. Overall, we achieve much higher scores. This class is easier to handle because there are more explicit keywords to indicate the relation. One notable subset of keywords are polar expressions (in particular if they are syntactically related to the health condition), however, bag of words can already pick up correlations between most polar expressions and this class. There are frequently occurring words, such as helfen (help), that are fairly unambiguous cues for this class.

UNSUIT displays similar properties as SUIT in that it also produces fairly low overall scores. Still, there is one feature that correlates fairly well with this class, namely the target food item being negated. Polar expressions, similar to SUIT, do not help. However, the learning curve also indicates further improvement given more training data.

Finally, CAUSE displays similar properties to BENEF. This class already produces high scores with a simple bag-of-words feature set. In addition, re-using a lexicon with cue words proposed for other datasets in previous publications also results in some notable improvement. Furthermore, a simple feature that indicates that the target food item has been mentioned prior to the health condition (in the sentence to be classified) also strongly correlates with that class. 
Acknowledgments The authors would like to thank Stephanie Köser for annotating the new resources presented in this article. We would also like to thank Benjamin Roth and Marc Schulder for interesting discussions. Michael Wiegand was partially supported by the German Federal Ministry of Education and Research (BMBF) under Grant No. 01IC12SO1X and the German Research Foundation (DFG) under Grant No. WI 4204/2-1.

\section{References}

Beamer, B., \& Girju, R. (2009). Using a bigram event model to predict causal potential. In Proceedings of the International Conference on Intelligent Text Processing and Computational Linguistics (CICLing) (pp. 430-441). Mexico City, Mexico.

Bunescu, R. C., \& Mooney, R. J. (2005). Subsequence kernels for relation extraction. In Proceedings of the Conference on Neural Information Processing Systems (NIPS). Vancouver, Canada.

Chahuneau, V., Gimpel, K., Routledge, B. R., Scherlis, L., \& Smith, N. A. (2012). Word salad: Relating food prices and descriptions. In Proceedings of the Joint Conference on Empirical Methods in Natural Language Processing and Computational Natural Language Learning (EMNLP/CoNLL) (pp. 1357-1367). Jeju Island, Korea.

Church, K. W., \& Hanks, P. (1990). Word association norms, mutual information, and lexicography. Computational Linguistics, 16(1), 22-29.

Cohen, A. M., \& Hersh, W. R. (2005). A survey of current work in biomedical text mining. Briefings in Bioinformatics, 6, 57-71.

Deng, L., \& Wiebe, J. (2014). Sentiment propagation via implicature constraints. In Proceedings of the Conference on European Chapter of the Association for Computational Linguistics (EACL) (pp. 377-385). Gothenburg, Sweden.

Deng, L., Wiebe, J., \& Choi, Y. (2014). Joint inference and disambiguation of implicit sentiments via implicature constraints. In Proceedings of the International Conference on Computational Linguistics (COLING) (pp. 79-88). Dublin, Ireland.

Diaz-Aviles, E., Stewart, A., Velasco, E., Denecke, K., \& Nejdl, W. (2012). Epidemic intelligence for the crowd, by the crowd. In Proceedings of the International AAAI Conference on Weblogs and Social Media (ICWSM). Dublin, Ireland.

Fisichella, M., Stewart, A., Cuzzocrea, A., \& Denecke, K. (2011). Detecting health events on the social web to enable epidemic intelligence. In Proceedings of the International Symposium on String Processing and Information Retrieval (SPIRE) (pp. 87-103). Pisa, Italy.

Gildea, D., \& Jurafsky, D. (2002). Automatic labeling of semantic roles. Computational Linguistics, 28(3), 245-288.

Girju, R. (2003). Automatic detection of causal relations for question answering. In Proceedings of the ACL 2003 Workshop on Multilingual Summarization and Question Answering (pp. 76-83). Sapporo, Japan.

Girju, R., \& Moldovan, D. (2002). Text mining and causal relations. In Proceedings of the International FLAIRS Conference (FLAIRS) (pp. 360-364). Pensacola Beach, FL, USA.

Girju, R., Badulescu, A., \& Moldovan, D. (2003). Learning semantic constraints for the automatic discovery of part-whole relations. In Proceedings of the Human Language Technology Conference (HLT) (pp. 80-87). Edmonton, Canada.

Hamp, B., \& Feldweg, H. (1997). GermaNet-A lexical-semantic net for German. In Proceedings of ACL Workshop Automatic Information Extraction and Building of Lexical Semantic Resources for NLP Applications (pp. 9-15). Madrid, Spain.

Joachims, T. (1999). Making large-scale SVM learning practical. In B. Schölkopf, C. Burges \& A. Smola (eds) Advances in kernel methods-Support vector learning (pp. 169-184). MIT Press.

Kessler, J. S., \& Nicolov, N. (2009). Targeting sentiment expressions through supervised ranking of linguistic configurations. In Proceedings of the International AAAI Conference on Weblogs and Social Media (ICWSM). San Jose, CA, USA.

Klenner, M., Petrakis, S., \& Fahrni, A. (2009). Robust compositional polarity classification. In Proceedings of Recent Advances in Natural Language Processing (RANLP) (pp. 180-184). Borovets, Bulgaria.

Kozareva, Z. (2012). Cause-effect relation learning. In Proceedings of the Text Graphs Workshop at ACL (pp 39-43). Jeju, Republic of Korea. 
Landis, J. R., \& Koch, G. G. (1977). The measurement of observer agreement for categorical data. Biometrics, 33(1), 159-174.

Miller, G., Beckwith, R., Fellbaum, C., Gross, D., \& Miller, K. (1990). Introduction to wordnet: an online lexical database. International Journal of Lexicography, 3, 235-244.

Mintz, M., Bills, S., Snow, R., \& Jurafsky, D. (2009). Distant supervision for relation extraction without labeled data. In Proceedings of the Joint Conference of the Annual Meeting of the Association for Computational Linguistics and the International Joint Conference on Natural Language Processing of the Asian Federation of Natural Language Processing (ACL/IJCNLP) (pp. 1003-1011). Singapore.

Moschitti, A., \& Basili, R. (2006). Complex linguistic features for text classification: A comprehensive study. In Proceedings of the European Conference in Information Retrieval (ECIR) (pp. 181-196). Sunderland, United Kingdom.

Munro, R., Gunasekara, L., Nevins, S., Polepeddi, L., \& Rosen, E. (2012). Tracking epidemics with natural language processing and crowdsourcing. In Proceedings of the Spring Symposium for Association for the Advancement of Artificial Intelligence (AAAI) (pp 52-58). Toronto, Canada.

Ng, V., Dasgupta, S., \& Arifin, S. M. N. (2006). Examining the role of linguistic knowledge sources in the automatic identification and classification of reviews. In Proceedings of the International Conference on Computational Linguistics and Annual Meeting of the Association for Computational Linguistics (COLING/ACL) (pp. 611-618). Sydney, Australia.

Popowich, F. (2005). Using text mining and natural language processing for health care claims processing. SIGKDD Explorations, 7(1), 59-66.

Rafferty, A., \& Manning, C. D. (2008). Parsing three German treebanks: Lexicalized and unlexicalized baselines. In Proceedings of the ACL Workshop on Parsing German (PaGe) (pp. 40-46). Columbus, $\mathrm{OH}, \mathrm{USA}$.

Roth, B., Barth, T., Wiegand, M., Singh, M., \& Klakow, D. (2014). Effective slot filling based on shallow distant supervision methods. In Proceedings of the Text Analysis Conference (TAC). Gaithersburg, MD: NIST.

Sokolova, M., \& Bobicev, V. (2011). Sentiments and opinions in health-related web messages. In Proceedings of Recent Advances in Natural Language Processing (RANLP) (pp. 180-184). Borovets, Bulgaria.

Torii, M., Yin, L., Nguyen, T., Mazumdar, C. T., Liu, H., Hartley, D. M., et al. (2011). An exploratory study of a text classification framework for internet-based surveillance of emerging epidemics. International Journal of Medical Informatics, 80(1), 56-66.

van Hage, W. R., Katrenko, S., \& Schreiber, G. (2005). A method to combine linguistic ontologymapping techniques. In Proceedings of International Semantic Web Conference (ISWC) (pp. 732744). Galway: Springer.

van Hage, W. R., Kolb, H., \& Schreiber, G. (2006). A method for learning part-whole relations. In Proceedings of International Semantic Web Conference (ISWC) (pp. 723-735). Athens, GA: Springer.

van Hage, W. R., Sini, M., Finch, L., Kolb, H., \& Schreiber, G. (2010). The OAEI food task: An analysis of a thesaurus alignment task. Applied Ontology, 5(1), 1-28.

Wiegand, M., \& Klakow, D. (2010). Convolution kernels for opinion holder extraction. In Proceedings of the Human Language Technology Conference of the North American Chapter of the ACL (HLT) NAACL) (pp. 795-803). Los Angeles, CA, USA.

Wiegand, M., \& Klakow, D. (2013a). Towards contextual healthiness classification of food items-A linguistic approach. In Proceedings of the International Joint Conference on Natural Language Processing (IJCNLP) (pp. 19-27). Nagoya, Japan.

Wiegand, M., \& Klakow, D. (2013b). Towards the detection of reliable food-health relationships. In Proceedings of the NAACL-Workshop on Language Analysis in Social Media (pp. 69-79). Atlanta, GA, USA.

Wiegand, M., Roth, B., \& Klakow, D. (2012a). Data-driven knowledge extraction for the food domain. In Proceedings of KONVENS (pp. 21-29). Vienna, Austria.

Wiegand, M., Roth, B., \& Klakow, D. (2012b). Web-based relation extraction for the food domain. In Proceedings of the International Conference on Applications of Natural Language Processing to Information Systems (NLDB) (pp. 222-227). Groningen: Springer.

Wiegand, M., Roth, B., Lasarcyk, E., Köser, S., \& Klakow, D. (2012c). A gold standard for relation extraction in the food domain. In Proceedings of the Conference on Language Resources and Evaluation (LREC) (pp. 507-514). Istanbul, Turkey. 
Wiegand, M., Roth, B., \& Klakow, D. (2014). Automatic food categorization from large unlabeled corpora and its impact on relation extraction. In Proceedings of the Conference on European Chapter of the Association for Computational Linguistics (EACL) (pp. 673-682). Gothenburg, Sweden.

Wilson, T., Wiebe, J., \& Hoffmann, P. (2005). Recognizing contextual polarity in phrase-level sentiment analysis. In Proceedings of the Conference on Human Language Technology and Empirical Methods in Natural Language Processing (HLT/EMNLP) (pp. 347-354). Vancouver, BC, Canada.

Witten, I., \& Frank, E. (2005). Data mining: Practical machine learning tools and techniques. San Francisco: Morgan Kaufmann Publishers. 